\title{
Applications and Major Achievements of Genome Editing in Vegetable Crops: A Review
}

\author{
Young-Cheon Kim ${ }^{1 \dagger}$, Yeeun Kang ${ }^{2 \dagger}$, Eun-Young Yang ${ }^{3}$, Myeong-Cheoul Cho ${ }^{3}$, \\ Roland Schafleitner ${ }^{4}$, Jeong Hwan Lee ${ }^{1}$ and Seonghoe Jang ${ }^{2 *}$
}

${ }^{1}$ Division of Life Sciences, Jeonbuk National University, Jeonju, South Korea, ${ }^{2}$ World Vegetable Center Korea Office, Wanju-gun, South Korea, ${ }^{3}$ National Institute of Horticultural and Herbal Science (NIHHS), Rural Development Administration (RDA), Wanju-gun, South Korea, ${ }^{4}$ World Vegetable Center, Tainan, Taiwan

\section{OPEN ACCESS}

Edited by:

Goetz Hensel,

Heinrich Heine University Düsseldorf,

Germany

Reviewed by:

Frank Hartung,

Julius Kühn-Institut, Germany

Ana Margarida Fortes,

University of Lisbon, Portugal

${ }^{*}$ Correspondence:

Seonghoe Jang

seonghoe.jang@worldveg.org

tThese authors have contributed equally to this work

Specialty section:

This article was submitted to

Plant Biotechnology,

a section of the journal

Frontiers in Plant Science

Received: 31 March 2021

Accepted: 18 May 2021

Published: 11 June 2021

Citation:

Kim Y-C, Kang Y, Yang E-Y,

Cho M-C, Schafleitner $R$,

Lee JH and Jang S (2021)

Applications and Major Achievements

of Genome Editing in Vegetable

Crops: A Review.

Front. Plant Sci. 12:688980.

doi: 10.3389/fpls.2021.688980
The emergence of genome-editing technology has allowed manipulation of DNA sequences in genomes to precisely remove or replace specific sequences in organisms resulting in targeted mutations. In plants, genome editing is an attractive method to alter gene functions to generate improved crop varieties. Genome editing is thought to be simple to use and has a lower risk of off-target effects compared to classical mutation breeding. Furthermore, genome-editing technology tools can also be applied directly to crops that contain complex genomes and/or are not easily bred using traditional methods. Currently, highly versatile genome-editing tools for precise and predictable editing of almost any locus in the plant genome make it possible to extend the range of application, including functional genomics research and molecular crop breeding. Vegetables are essential nutrient sources for humans and provide vitamins, minerals, and fiber to diets, thereby contributing to human health. In this review, we provide an overview of the brief history of genome-editing technologies and the components of genome-editing tool boxes, and illustrate basic modes of operation in representative systems. We describe the current and potential practical application of genome editing for the development of improved nutritious vegetables and present several case studies demonstrating the potential of the technology. Finally, we highlight future directions and challenges in applying genomeediting systems to vegetable crops for research and product development.

Keywords: CRISPR-Cas application, genome-editing technology, precision breeding, transformation, vegetables

\section{INTRODUCTION}

There has been growing interest in the beneficial effect of consuming vegetables because of the broad range of nutritional compounds, such as vitamins, minerals, antioxidants, dietary fiber, and a plethora of phytochemical compounds present in this crop group (Septembre-Malaterre et al., 2018). While vitamins and minerals are essential nutrients for humans, antioxidant compounds from fruits and vegetables are known to reduce cellular oxidative stress and the risk of chronic disease, like diabetes, cancer, and cardiovascular disease (Serafini et al., 2002; Ninfali et al., 2005; Aune et al., 2017; Miller et al., 2017). However, vegetables, like all other crops, are generally sensitive to biotic and abiotic stresses, and thus, disease, high temperature, 
and limited water supply are major limiting factors in vegetable productivity. These factors will be further magnified by climate change (Bisbis et al., 2018). Therefore, researchers are striving to improve vegetable varieties in terms of yield and yield stability, nutritional value, and biotic and abiotic stress tolerance by classical breeding and by using plant molecular breeding technologies (Abdallah et al., 2015).

Plant breeding is a complex process through which new crop varieties with desirable characteristics are developed and strategies are devised to combine these characteristics to obtain superior varieties (Glenn et al., 2017). The first step in breeding is to make use of genetic variation between individuals within a plant species. For decades, this has been achieved by crossing parental material or by applying physical or chemical mutagenesis to crop plants (Holme et al., 2019; Kleter et al., 2019). Since pioneering research at the beginning of the twentieth century detected the capacity of X-rays to change the plant phenotype in crops, such as barley and maize, a series of novel mutagenesis breeding tools have been developed (Muller, 1927; Stadler, 1928a,b). More sophisticated radiation techniques, such as gamma radiation, UV light, and particle radiation, were then developed to generate novel agronomic traits in crops and to study gene functions (Shu et al., 2012). Later, mutagenic chemicals were preferred for mutagenesis because of easier handling and higher mutation frequency compared to radiological methods. Today, the most widely used chemical mutagen is ethyl methanesulfonate, with other chemical mutagens, such as sodium azide and methyl nitrosourea (Az-MNU), also in frequent use for mutagenesis in plants (Till et al., 2004, 2007).

Physical and chemical mutagenesis has been successfully employed to induce point mutations and deletions in the plant genome (Jankowicz-Cieslak et al., 2017), and thousands of varieties, derived from mutation breeding, have been released (Ahloowalia et al., 2004). The promise of mutagenesis in vegetable breeding has remained largely unfulfilled, but it is widely used to generate variation in ornamentals (Bernardo, 2016). As molecular technologies and DNA sequencing technologies advanced, researchers began to study the functional characteristics of genes by using T-DNA-tagged mutant pools generated by random T-DNA insertional mutagenesis, enabling identification of DNA sequences flanking T-DNA to explore potential genetic elements responsible for phenotypic alterations in mutants. The information accumulated from the analyses of T-DNA flanking sequences is one of the stepping stones that provided new pathways for producing improved crop varieties harboring desired traits with the aid of molecular breeding tools (Radhamony et al., 2005; Chaudhary et al., 2019; Kleter et al., 2019).

There have been a number of major advances in molecular biological methods over the last few decades. The discovery of sequence-specific nucleases (SSNs) and the development of the clustered regulatory interspaced short palindromic repeats (CRISPR)/CRISPR-associated protein (Cas) system have enabled programmable gene editing at the DNA level to generate vegetables with altered functions and desired traits (Abdallah et al., 2015; Nunez de Caceres Gonzalez and De la Mora Franco, 2020).
In this review, we describe the brief history of genome editing, the components of the genome-editing tool boxes, and basic modes of gene-editing systems. In addition, we present examples of practical application of those tools in editing vegetable genomes. Finally, future directions and challenges associated with genome-editing systems for the production of vegetables with desired traits are discussed.

\section{HISTORY OF GENOME-/GENE-EDITING TECHNOLOGY}

Over the past few years, the development of gene-/genomeediting technologies has facilitated the precise and efficient targeted modification of genomes in various organisms, including vegetable crops to increase yield and quality (Chen and Gao, 2014). Advanced molecular biological methods using SSNs, such as zinc-finger nucleases (ZFNs) and transcription activatorlike effector nucleases (TALENs), and CRISPR-Cas system (Kim et al., 1996; Christian et al., 2010; Jinek et al., 2012; Chen and Gao, 2014; Gao, 2014), have made it possible for plant researchers to conduct targeted gene/genome engineering precisely and efficiently. These techniques generate double-strand breaks (DSBs) at specific DNA sites and, via the endogenous DNA repair system, induce insertions or deletions of nucleotides by non-homologous end joining (NHEJ), or cause gene replacements by homologous recombination (HR) thereby resulting in loss-of-function or gain-of-function of target genes (Symington and Gautier, 2011; Figure 1). The various CRISPR-Cas systems have been revealed to be powerful tools for gene/ genome editing, and numerous genome-edited plants have been created by these gene-/genome-editing technologies (Gao, 2014).

\section{Zinc-Finger Nucleases}

Zinc-finger nucleases, the first generation of site-specific nucleases, allowed the rapid and targeted modification of the genome (Kim et al., 1996). ZFNs are typically generated by fusing zinc-finger protein (ZFP) domains and are capable of sequence-specific DNA binding, with a nonspecific DNA cleavage domain from bacterial FokI endonuclease (Figure 1A; Petolino, 2015). Each ZFP with a tandem array of cysteine 2 and histidine 2 $\left(\mathrm{Cys}_{2}-\mathrm{His}_{2}\right)$ domains (Miller et al., 1985) recognizes approximately 3 bp of DNA sequence. Generally, four to six ZFPs are linked together to recognize a specific DNA sequence (12-18 bp; Urnov et al., 2010). The FokI catalytic domain must dimerize for DNA cleavage (Bitinaite et al., 1998), so ZFNs are used as heterodimers to target and cut the DNA. Once the zincfinger domain recognizes and binds the target DNA, the fused FokI nuclease domain cleaves the DNA sequence, inducing DSBs at their target locus (Weinthal et al., 2010; Lee et al., 2016).

Zinc-finger nucleases have been employed to modify the targeted gene sequence in several crops, such as maize (Shukla et al., 2009; Ainley et al., 2013), soybean (Curtin et al., 2011), rice (Cantos et al., 2014; Jung et al., 2018), and apple (Peer et al., 2015). As a representative example of the genome editing in crops by ZFNs, Shukla et al. (2009) reported the precise 
A

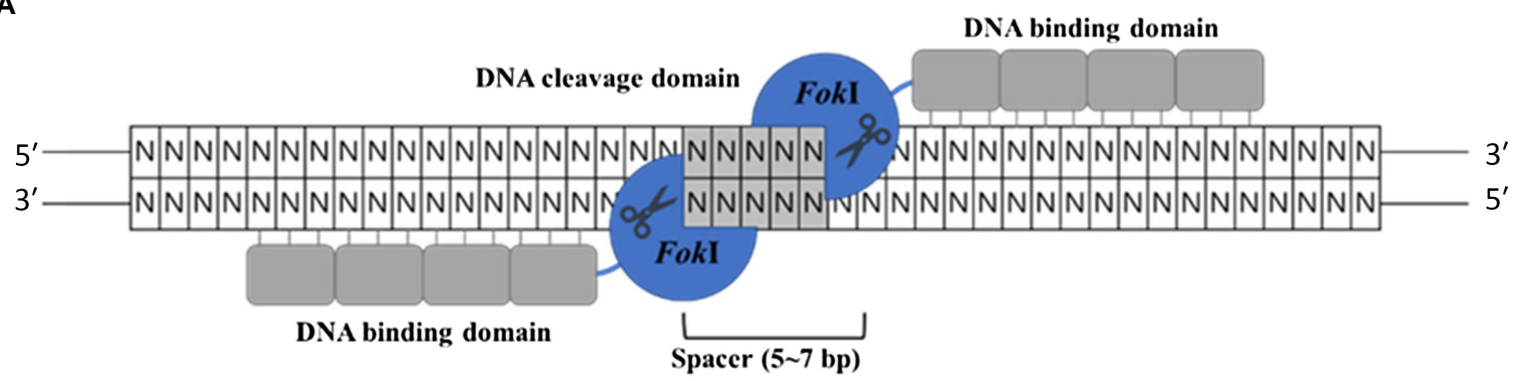

B

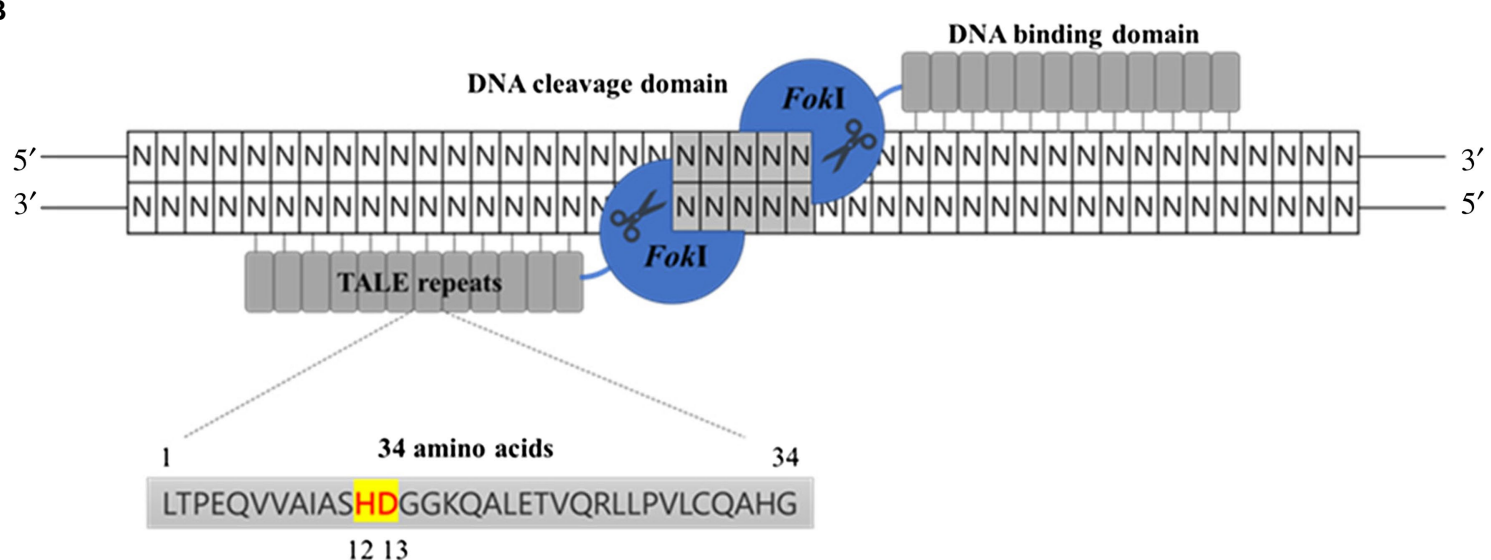

C

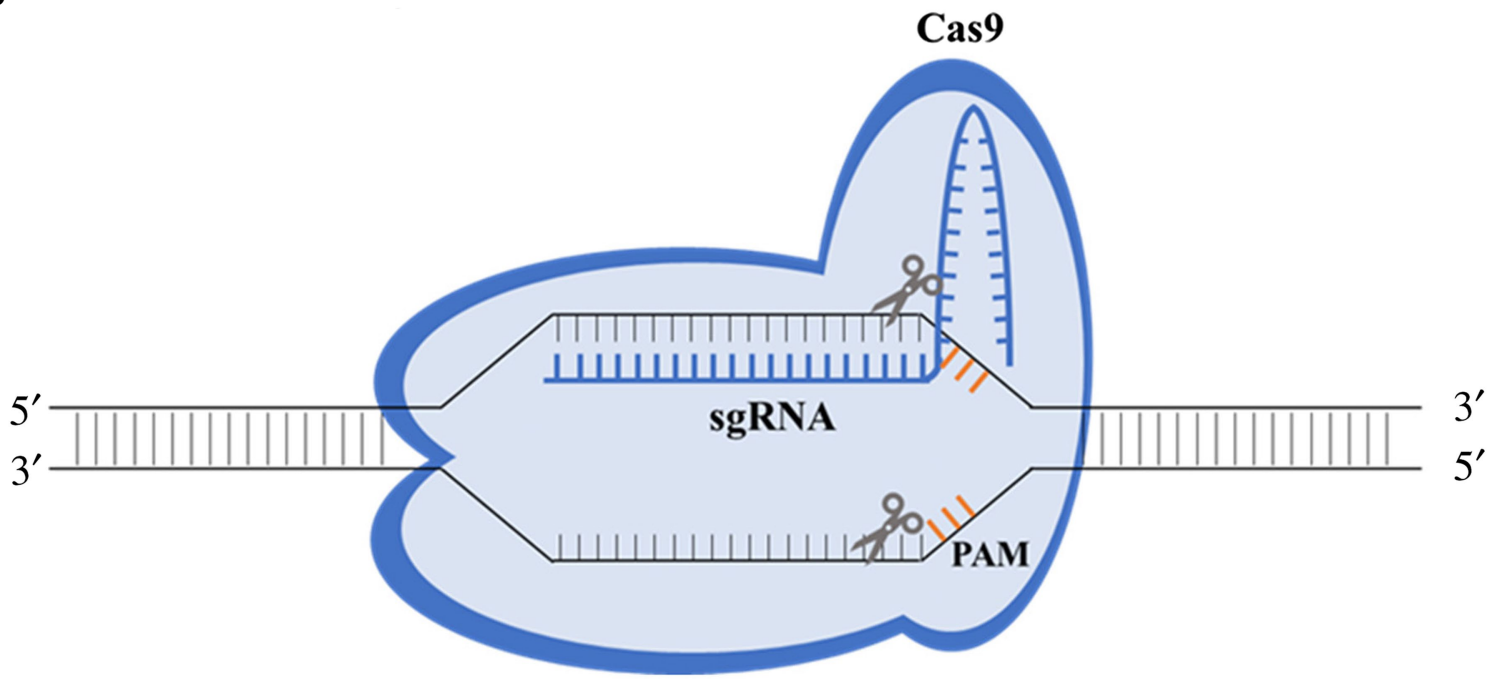

FIGURE 1 | Some of the major genome-editing technologies using site-specific nucleases include zinc-finger nucleases (ZFNs), transcription activator-like effector nucleases (TALENs), and the clustered regularly interspaced short palindromic repeats/CRISPR-associated 9 (CRISPR-Cas9) systems. (A) The ZFN-binding domain is comprised of multimerized zinc-finger proteins (ZFPs). Each ZFP recognizes approximately 3 bp of DNA sequence, and the fused Fokl nuclease domains dimerize and generate double-strand breaks. (B) Like ZFNs, TALENs consist of a DNA-binding domain, termed transcription activator-like effector (TALE) repeats, and nuclease domain of Fokl enzyme. Each TALE repeat consists of a highly conserved 34-amino acid sequence with hypervariable twelfth and thirteenth amino acids, which allow the recognition of the single nucleotide. (C) In the CRISPR-Cas9 system, a single-guide RNA (sgRNA) guides the Cas9 nuclease to direct the cleavage of cognate DNA sequences adjacent to 5'-NGG-3' protospacer-adjacent motifs (PAMs).

targeted insertion of the PAT herbicide-tolerance gene at the IPK1 locus in maize (Shukla et al., 2009). These mutated maize plants exhibited herbicide tolerance as well as altered inositol phosphate profiles in the developing seed. ZFNs were also used for the elucidation of functional roles of the SSIVa encoding an isoform of soluble starch synthase in rice starch biosynthesis (Jung et al., 2018). Transgenic plants containing ZFN-induced mutations at the SSIVa locus showed dwarfism with reduced 
starch content. The modularity of ZFNs is a major advantage for the design of DNA-binding proteins that can recognize a broad spectrum of DNA sequences for genome editing (Bhakta et al., 2013). Despite the advantage of ZFNs in genome editing, several inherent disadvantages have restricted their wide range of application, such as expensive and time-consuming processes for optimized assembly of the ZF domain to bind DNA with high affinity and low frequency in selection of target sites, which can only be used every 200 bps in DNA sequences and occurrence of imperfect modular structure in zinc-finger assembly and/or nonspecific binding of the FokI cleavage domain, which can raise the risk of off-target effects and/or cellular toxicity (Eid and Mahfouz, 2016; Mushtaq et al., 2018).

\section{Transcription Activator-Like Effector Nucleases}

Transcription activator-like effector nucleases are the second generation of site-specific nucleases, which have emerged as an alternative to ZFNs for genome editing (Sakuma and Yamamoto, 2017). TALENs consist of DNA-binding and nuclease domains of FokI enzyme like ZFNs. The DNA-binding domain, termed transcription activator-like effector (TALE) repeats, is typically composed of repetitive sequences of residues derived from TALEs secreted by Xanthomonas bacteria to aid the infection of plant species (Figure 1B). Each DNA-binding repeat recognizes a single nucleotide of the genomic sequence (Boch and Bonas, 2010; Joung and Sander, 2013). An individual DNA-binding repeat consists of a highly conserved 34-amino acid sequence with a highly polymorphic region at positions 12 and 13, which are referred to as the repeat variable di-residue (RVD) determining the nucleotide-binding specificity (Richter et al., 2020). Therefore, a TALE DNA-binding domain with the nuclease domain of FokI can generate DSBs at a desired target site in the genome by selecting a combination of RVDs. Like ZFNs, TALEN-mediated genome editing has achieved targeted mutagenesis in a variety of crop species, such as rice (Li et al., 2012; Shan et al., 2013), soybean (Haun et al., 2014), wheat (Wang et al., 2014), and potato (Sawai et al., 2014). Two seed-specific genes, FAD2-1A and FAD2-1B encoding a fatty acid desaturase-2 enzyme (FAD2-1) that converts oleic acid to linoleic acid in soybean, were deleted to improve the quality of soybean seed oil by TALENs system (Haun et al., 2014). The genetically engineered soybean plants produced nearly four times more oleic acid than the wild-type (WT) parents. In addition, Wang et al. (2014) successfully utilized the TALENs genome-editing system in wheat to conduct the targeted manipulation of three $M L O$ loci, which encode proteins that repress defenses against powdery mildew diseases in other plants: TALEN-induced tamlo-aabbdd wheat plants showed strong resistance to powdery mildew (Wang et al., 2014).

A TALE repeat module recognizes a single nucleotide, while a single zinc-finger module recognizes three nucleotides of DNA (Jankele and Svoboda, 2014), so the design of the TALEN genome-editing machinery is more facile and adaptable compared to ZFNs. In addition, in contrast to ZFNs, the TALE repeat array can be extended to any desired length, though the use of larger TALENs may result in less specificity (Gupta and Musunuru, 2014). Although TALEN has the advantage of greater flexibility, it is sensitive to cytosine methylation especially at CpG dinucleotides (Valton et al., 2012), and to be active, it requires thymine before the $5^{\prime}$ end of the target sequence, which is recognized by two amino-terminal cryptic repeat folds (Kim and Kim, 2014).

\section{CRISPR-Cas9}

Even though both ZFN and TALEN have been proven to be quite effective for gene editing, these tools are relatively expensive and laborious to engineer, which has restricted their application (Gaj et al., 2013; Erpen-Dalla Corte et al., 2019). DNA/RNA-mediated adaptive immune systems involving type II CRISPR and Cas immunity in bacteria and archaea that protect against invading plasmids and viruses provide an alternative genome-editing strategy (Barrangou, 2013). In this system, when foreign DNA from a virus or plasmid is integrated into a CRISPR locus, the CRISPR repeat-spacer array is converted into the mature CRISPR RNA (crRNA). The mature crRNA that is base-paired to trans-activating crRNA (tracrRNA) guides the CRISPR-associated endonuclease, Cas9 to cleave the protospacer DNA on both strands of the invader (Horvath and Barrangou, 2010; Jinek et al., 2012). The CRISPR-Cas9 mechanism has emerged as a powerful universal genome-editing tool, also for targeted trait improvement in crops (Arora and Narula, 2017).

The CRISPR-Cas9 system for genome editing has two components: the Cas9 nuclease and a single-guide RNA (sgRNA) consisting of the artificial fusion of a crRNA and the scaffold tracrRNA (Figure 1C; Jinek et al., 2012). The sgRNA guides Cas9 to direct the cleavage of cognate DNA sequences predominantly $3 \mathrm{bp}$ away from the 5'-NGG-3' protospaceradjacent motif (PAM; Fischer et al., 2012). Therefore, a variety of DNA sites can be targeted by Cas9 through exchange of the 20-bp spacer sequence in the guide RNA (gRNA) with a sequence that is complementary to the target site (Cong et al., 2013). Major advantages of the CRISPR-Cas9 system over ZFNs and TALENs are simplicity and the low cost of engineering of the system, its adaptability to virtually to any target region with a PAM sequence nearby, and its capacity for multiplexing, meaning that multiple sites can be targeted for mutagenesis simultaneously by using multiple sgRNAs while expressing a single Cas9 nuclease gene (Pennisi, 2013; Minkenberg et al., 2017). However, there are still some challenges to be tackled to apply the CRISPR-Cas9 technology to the gene/genome editing of crops. Although the CRISPR-Cas9 system is more precise and efficient in gene/genome editing than other previously developed genetic engineering methods, there is a risk of off-target effects, which are defined as unintended cleavages and/or mutations at untargeted genomic sites (Zhao and Wolt, 2017). Off-target mutations may cause unintended effects, especially in species with large and complex genomes (Alkan et al., 2018). Methods for reducing the risk of off-target mutations have been developed, as discussed below. Furthermore, off-target effects in plants can be mitigated by selecting the desired 
phenotypes in the pool of mutated individuals or by breeding. CRISPR-Cas9 is thought to be particularly advantageous in plant breeding to change a single or a few alleles in a line, especially when the alternative would consist of introgressing the desired allele from non-adapted accessions or wild species. In these cases, CRISPR-Cas9-mediated gene editing avoids linkage drag and makes backcrossing to achieve the recurrent phenotype unnecessary. It allows producing specific mutations effectively in a selected genetic background.

\section{PRECISE GENOME EDITING IN PLANTS}

Targeted mutagenesis in plants relies on NHEJ to repair DSBs, which is error-prone and, at a certain frequency, results in small deletions, insertions, or nucleotide substitutions (Atkins and Voytas, 2020). Homology-directed repair (HDR)-mediated repair is less common than the NHEJ repair pathway due to very low editing efficiency achieved with $\mathrm{HDR}$, but allows targeted gene insertions or replacement, if a repair template is provided. The modern mutation tools, including ZNFs,
TALENs, and CRISPR-Cas systems, have revolutionized the field of plant molecular biology and functional genomics research and have the potential to facilitate crop development by altering agronomic traits (Chen et al., 2019). Although the ZNF and TALEN systems have contributed to the targeted mutagenesis studies in a variety of plant species, such as Arabidopsis, maize, Brassica, rice, barley, soybean, tobacco, tomato, wheat, potato, and sugarcane (Shelake et al., 2019), the CRISPR-Cas system is now the most widely adopted tool for genome editing due to its simple engineering process, versatility, low cost, high efficiency, and high specificity (Brandt and Barrangou, 2019) and has great potential for genome editing to develop valueadded improved cultivars (Zhang et al., 2020c). In the following sections, we provide an overview of the most commonly used and the newly developed CRISPR-Cas9 technologies for precise genome editing in plants.

\section{Basic CRISPR-Cas System}

The CRISPR-Cas9 system has been widely adopted as a genome engineering platform (Figure 2A). Cas9 (SpCas9), the central player in the type II CRISPR-Cas Class2 system of Streptococcus

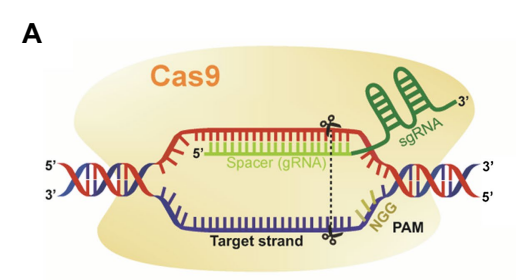

B

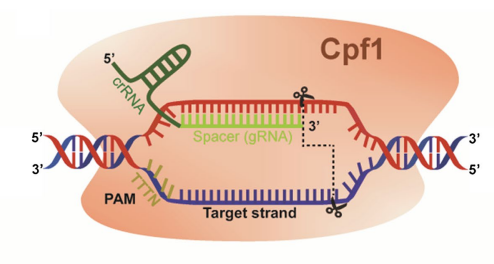

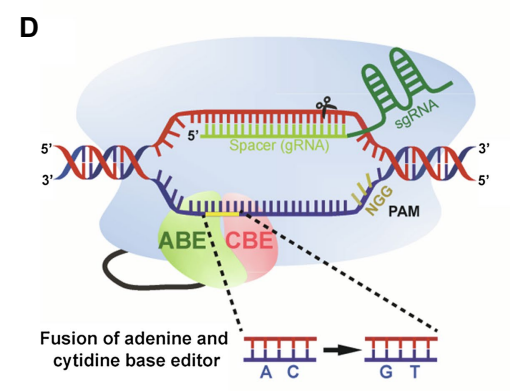

E

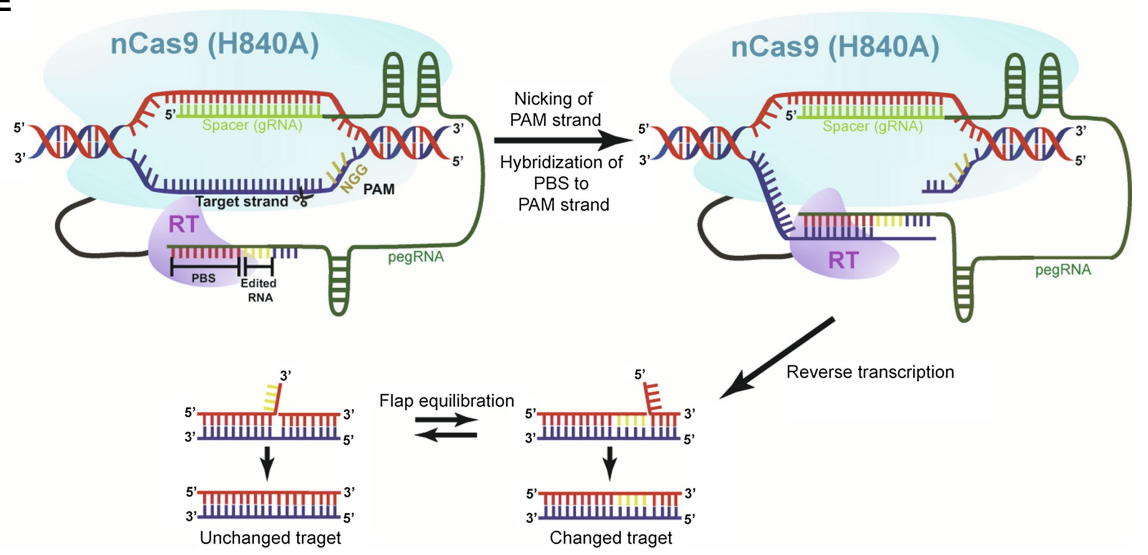


pyogenes, was the first nuclease harnessed for genome editing and is still the most commonly used enzyme for this purpose (Cong et al., 2013; Mali et al., 2013). A sgRNA is designed to function in the crRNA-tracrRNA complex. Cas9 protein can be targeted to specific genomic loci by sgRNAs with various sequences: Cas9 recognizes sgRNAs and is then guided to bind a complementary sequence at the target sites, which is positioned next to PAM by hybridization of the spacer part of the sgRNAs. Subsequently, the CRISPR-Cas9 system breaks both strands of the DNA, resulting in blunt end cuts generally three base pairs upstream $(-3 \mathrm{bp})$ of the PAM site (5'-NGG; Gasiunas et al., 2012; Jinek et al., 2012; Makarova et al., 2015).

To expand the limited availability of SpCas9 target sites by recognizing different PAM sequences, additional SpCas9 variants have been developed, such as $\mathrm{xCas} 9$ recognizing $5^{\prime}-\mathrm{NG}$, 5'-GAA, and 5'-GAT (Hu et al., 2018), Staphylococcus aureus Cas9 (SaCas9) recognizing 5'-NNGRRT (Ran et al., 2015), S. thermophilus Cas9 (StCas9) recognizing 5'-NNAGAAW (Cong et al., 2013), Neisseria meningitidis Cas9 (NmCas9) recognizing 5'-NNNNGATT (Esvelt et al., 2013), Campylobacter jejuni Cas9 (CjCas9) recognizing 5'-NNNVRYM (Yamada et al., 2017), and CasX known as Cas12e recognizing 5'-TTCN (Burstein et al., 2017). Cas13 variants mediating high interference activities against target RNA viruses have been also applied to plants for studying virus biology (Mahas et al., 2019).

After a DSB is inserted at the target site by CRISPR-Cas9, DNA repair occurs via NHEJ, which often causes mutations, such as an insertion or more often a deletion at the repair site. If this mutation is in a genic region, such indels very likely generate a premature stop codon downstream of the target sites (Cong et al., 2013; Mali et al., 2013). Cpf1 known as Cas12a, another Class 2 endonuclease, is similar to Cas9 in size and shape, and recognizes 5 -TTTTN or TTTN as the PAM sequence (Zetsche et al., 2015). However, functional Cpf1 does not require tracrRNA - but still needs gRNA providing specificity. In addition, Cpf1 generates a staggered cut with a 5 ' overhang at the target sites, which may be beneficial for the correct orientation of integrating DNA (Figure 2B). Furthermore, AsCpf1, a Cpf1 orthologue from Acidaminococcus spp., recognizes the 5'TTTV as a PAM sequence and their variants were recently engineered to increase genome-editing activity and expand PAM recognition sites by recognizing 5'-TYCV and 5'-TATV or 5'-VTTV, 5'-TTTT, 5'-TTCN, and TATV (Gao et al., 2017; Kleinstiver et al., 2019).

\section{Base Editing}

Deaminase-mediated base editing technology is an alternative genome-editing tool, which can generate precise point mutations in the target region of genomes without making DSBs. Instead, a base editor is fused to a Cas9 nickase and is targeted to a specific DNA sequence with a gRNA. After the advent of two primary base editing tools, cytosine base editors (CBEs) and adenine base editors (ABEs; Shimatani et al., 2017; Li et al., 2020b), dual base editor-mediated precise genomeediting technology was developed in plants (Li et al., 2020b).
CBE consists of a Cas9 nickase (nCas9) harboring the D10A mutation that inactivates the RuvC, one of the two nucleolytic domains (RuvC and $\mathrm{HNH}$ ) of Cas9, fused with a cytidine deaminase and uracil glycosylase inhibitor (UGI; Figure 2C). Deamination on cytidine catalyzed by cytidine deaminase in the targeting strand DNA region converts the cytidine into uridine. When nCas9 (D10A) protein nicks the nontargeting DNA strand, the induced U-G mismatch generates T-A in the resulting DNA strands through the DNA repair and replication processes (C-to- $\mathrm{T}$ ). CBE-mediated base editing technology has been used in various crops, such as rice, maize, wheat, and potato with relatively high efficiency (Shimatani et al., 2017; Zong et al., 2017; Ren et al., 2018; Zong et al., 2018; Jin et al., 2020).

Adenine base editor, another base editing technology, expands base editing by converting A-T to G-C. The deamination of adenosine by adenosine deaminase fused with nCas9 (D10A) yields inosine, which can be paired with cytidine and is also recognized as guanine by DNA polymerase during DNA repair and replication (Gaudelli et al., 2017). Although ABE variants were developed in Arabidopsis (Kang et al., 2018), wheat, rice, and rapeseed (Li et al., 2018a), the system showed lower efficiency than the original SpCas9 or SaCas9 (Hua et al., 2019; Li et al., 2020b). Several ABE variants recently developed for adenine base editing in mammalian cells may be useful for efficient editing in plants (Gaudelli et al., 2020; Richter et al., 2020).

Dual base editing technology converting C-G to T-A and A-T to G-C simultaneously is based on the fusion of nCas9 with the two base editors mentioned above, which is referred to as saturated targeted endogenous mutagenesis editor (STEME). It generates simultaneous dual base substitutions (C-G to T-A and A-T to G-C) with a sgRNA, which was recently used for genome editing in rice (Li et al., 2020b). The STEME system composed of an ABE and a CBE, nCas9 (D10A), sgRNA, and a UGI facilitates directed evolution of endogenous genes (Figure 2D; Li et al., 2020b). Given that STEMEs can generate diverse mutations, including base substitutions and in-frame indels with high efficiency, dual base editing might be applicable for a study on cis-elements of noncoding regions and even genome-wide screening of cis-regulatory regions.

\section{Prime Editing}

One of the major limitations of current genome-editing tools is in the technical difficulty with extremely low efficiency for introduction of customized sequences at target sites. Anzalone et al. (2019) developed the revolutionary genomeediting technology known as prime editing to solve this challenge (Anzalone et al., 2019). Prime editing enables 12 kinds of base conversions to target genes at locations ranging from 3 bp upstream to 29 bp downstream of PAM, consisting of precise insertions of up to $44 \mathrm{bp}$, and deletions of up to $80 \mathrm{bp}$ without inducing DSBs (Anzalone et al., 2019). The prime editing system is composed of a nCas9 (H840A) fused to a reverse transcriptase (RTase) and a prime editing 
guide RNA (pegRNA). The pegRNA driving nCas9 (H840A) to bind the target sequence is comprised of two regions: One is the spacer complementary to the sequences of the nonedited DNA strand at 5' of the pegRNA and the other region is located at the $3^{\prime}$ of the pegRNA containing prime binding site (PBS) required for the recognition of the sequences of DNA strand to be edited and the desired sequences, which will be introduced into the target site (Figure 2E). The PBS region plays a role as a primer for the RTase linked to the nCas9 (H840A). The RTase uses the pegRNA as the template, which pairs with the nCas9 (H840A)-nicked ssDNA strand, thereby resulting in a direct copy of the genetic information from the pegRNA into the target genome site (Anzalone et al., 2019). After the reverse transcription, the equilibration between 3' edited DNA flap and 5' unedited DNA flap occurs followed by integration of edited DNA into the target site of the genome via ligation and DNA repair system (Anzalone et al., 2019). Despite the prime editing system being a powerful genome-editing tool enabling the generation of 12 base substitutions and indels in rice and wheat, their practical efficiency in plants is limited, and also its capability for precise gene editing, up to now, was verified only in the two food crops: rice and wheat (Lin et al., 2020). Thus, more case studies demonstrating the applicability of the prime editing technology in various organisms are needed to accelerate the use of prime editing tools, and make steady progress toward technology improvement.

\section{Chromosome Engineering}

The aim of breeding is to combine as many favorable traits as possible in a breeding line. Traits are conditioned by genes, and the genes are located on chromosomes. Genes located close to each other are almost always inherited together, a relationship referred to as complete linkage. Sometimes, target genes can be tightly linked with unfavorable traits and this linkage may be difficult to break through classical breeding. It was suggested that induction of cross overs (COs) to separate unfavorable linkages can be achieved by CRISPR-Cas9 and subsequent HR as a consequence of induced DSBs (Filler Hayut et al., 2017) although the frequency of these COs is low, and it requires a selection system that detects these HR events efficiently. Early studies showed that CRISPR-Cas9-mediated DSB induction enabled DNA fragment inversions in plants (Gao et al., 2015; Zhang et al., 2017) and CO suppression as large inversions could be eliminated successfully by reverting the inversion (Giraut et al., 2011), making the region again accessible to meiotic COs (Huang and Puchta, 2021). Introduction of multiple DSBs, with low efficiency, is also able to cause inversions (Schmidt et al., 2019). Schmidt et al. (2020) showed that a 1.1 Mb inversion on chromosome 4 of Arabidopsis could be reversed using SaCas9 for DSB induction (Schmidt et al., 2020). What is more, heritable CRISPR-Cas9-mediated reciprocal translocations in the $\mathrm{Mbp}$ range were obtained between different nonallelic chromosomes (Beying et al., 2020). This approach provides a new method for plant breeding: Introgression of genes from a wild relative in a cultivar may be strongly enhanced by reducing linkage drag and making lengthy backcrossing unnecessary.

\section{DELIVERY OF CRISPR-CAS AGENTS IN PLANTS}

Through the rapid evolution of CRISPR-Cas tools with various functionalities, capabilities, and specialized application, CRISPRCas-mediated plant genome editing has become a very efficient and effective application in the area of crop improvement and translational research (Vats et al., 2019). However, there are bottlenecks in application of the editing technology to crop functional genomics and crop improvement. Researchers have focused on the delivery of gene-/genome-editing reagents to plant cells, which is one of the critical bottlenecks for successful plant gene/genome editing to produce the intended effects. The most commonly used delivery methods for DNA constructs carrying the CRISPR-cas9 components are biolistic bombardment, Agrobacterium-mediated delivery to explants or plants, and direct transfer of the constructs to protoplasts with polyethylene glycol (PEG), which have been successful for generating CRISPRCas-mediated genome editing in various plant species with varying efficiencies of transformation (Borrelli et al., 2018). However, these conventional delivery methods (plasmid-based transfer) have limitations, such as low efficiency and/or difficulty in transformation and regeneration depending on the plant species or genotype. Together with these limitations, conventional delivery methods also have controversy about safety concerns and legal restrictions on the use of recombinant DNA, warranting the development of DNA-free gene-editing methods.

A delivery method of the preassembled CRISPR-Cas9 ribonucleoproteins (RNPs) into protoplasts has been considered as an approach for DNA-free genome editing (Woo et al., 2015; Lee et al., 2016). CRISPR-Cas9 RNP-mediated genome editing has been reported in Arabidopsis, tobacco, lettuce, rice, maize, and bread wheat (Woo et al., 2015; Svitashev et al., 2016; Liang et al., 2017). Nevertheless, after direct CRISPR-Cas9 RNP delivery to protoplasts by PEG-mediated transfection, the process of protoplast regeneration is laborious, sophisticated, and time consuming and it is highly challenging to obtain whole regenerated plants (Figure 3A).

Due to the limitations of delivering CRISPR-Cas9 riboprotein complexes to plant cells, Agrobacterium-mediated T-DNA transformation is one of the most widely used delivery methods, allowing T-DNA cassettes including CRISPR-Cas machinery to be transferred into intact plant cells or explants (Figure 3A). Although biolistic bombardment also can deliver T-DNA to plant cells, it is limited by low transformation efficiency and can cause chromosome damage and trigger a range of DNA rearrangement processes (Liu et al., 2019). The T-DNA cassette can also be transferred into protoplasts by PEG-mediated transfection or electroporation. Alternatively, magnetofection and electroporation transformation methods transferring the T-DNA cassette to microspore and pollen have been used for cotton and wheat genome editing (Figure 3A) although the transformation efficiency is extremely low (Zhao et al., 2017; 


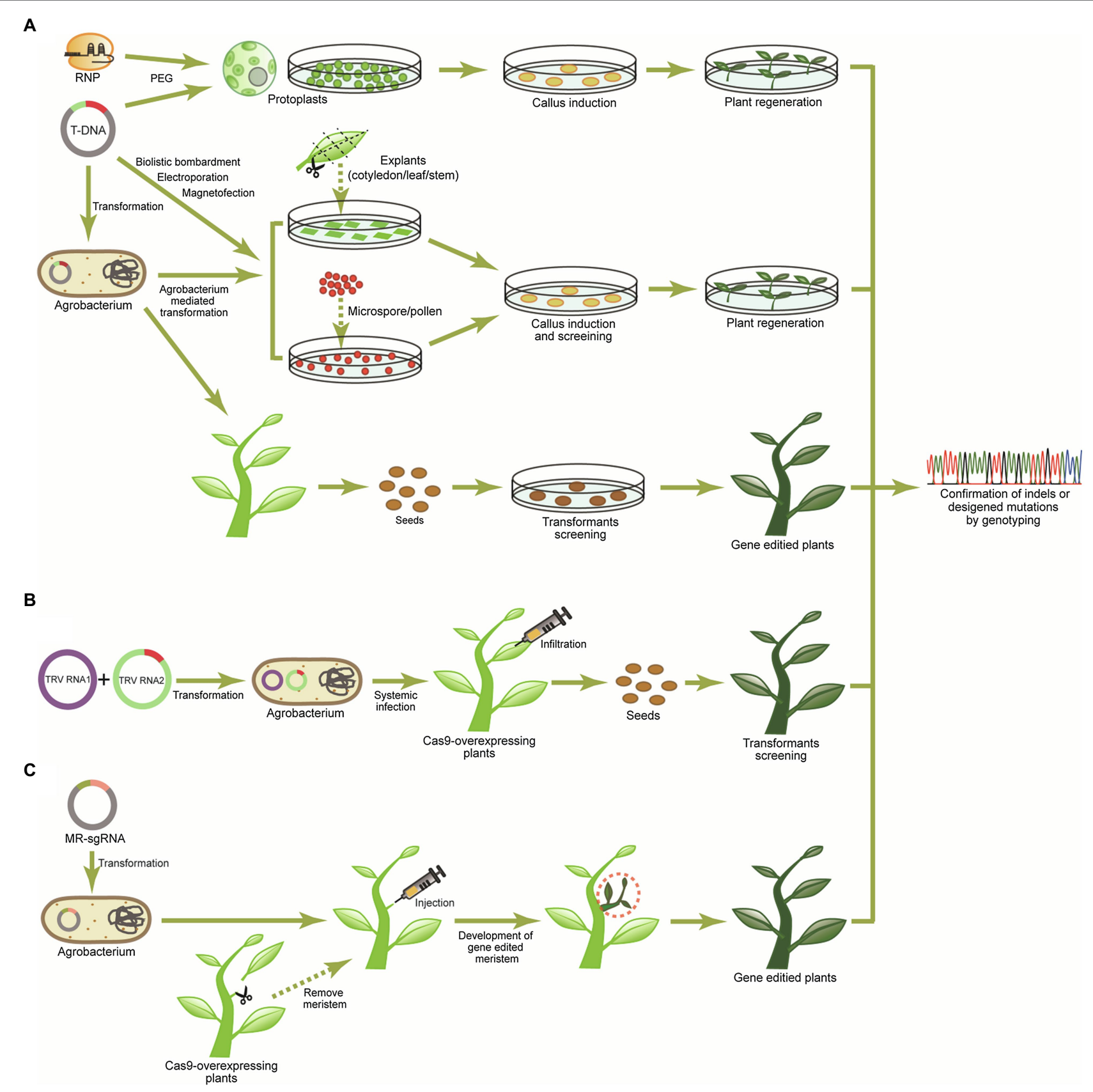

FIGURE 3 | Strategies for delivery of the CRISPR-Cas system into plants. (A) The preassembled CRISPR-Cas9 ribonucleoproteins (RNPs) can be delivered into protoplasts through the polyethylene glycol (PEG)-mediation, and T-DNA encoding CRISPR-Cas reagents [Cas protein and sgRNA(s)] can be delivered into the rigid plan cells (explants, microspores/pollens, and intact plants) using Agrobacterium-mediated transformation, biolistic bombardment, and magnetofection. Subsequently, regeneration procedures of protoplasts and tissues carrying CRISPR-Cas reagents are needed to produce genome edited lines. (B) In the virusinduced gene-editing system, sgRNA fused with RNA mobile element is integrated into tobacco rattle virus (TRV) RNA2. After transformation of TRV RNA1 and TRV RNA2 to Agrobacterium, infiltration is conducted to Cas9-overexpressing plants resulting in systemic spreading of the sgRNA by the mobile elements and induction of mutagenesis. (C) For de novo meristem induction system, the meristems of Cas9-overexpressing plants are removed for infiltration, and then, Agrobacterium carrying morphogenic regulators (MRs) and sgRNA are injected into pruning sites. MRs induce the de novo gene-edited meristem, and the gene-edited plants can finally be obtained from newly developed shoots.

Bhowmik et al., 2018; Vejlupkova et al., 2020). Recently, simple and high-throughput T-DNA binary vector cloning systems, which can express CRISPR-Cas reagents, including SpCas9 and a sgRNA or multiplexed gRNAs under the control of Cauliflower Mosaic Virus (CaMV) $35 S$ and U6/U3 promoters, have been developed for plant genome editing (Kim et al., 2016; 
Oh et al., 2020), and transformation efficiencies have been increased using nanoparticles to deliver DNA to plant cells (Jat et al., 2020). However, regeneration of transformants remains a limiting factor.

Plant virus-mediated genome editing is highly efficient and avoids the tissue regeneration steps since viruses replicate and systemically move around in plants (Figure 3B). However, because the CRISPR-Cas9 system is too large to be integrated into the genome of viruses commonly used for plant gene function studies and/or to be delivered into plant cells by infection of viruses, virus-mediated genome editing in plants should be conducted in Cas9-overexpressing plants. Singlestranded RNA viruses, such as tobacco rattle virus (Ali et al., 2015, 2018), tobacco mosaic virus (TMV; Cody et al., 2017), pea early-browning virus (PEBV; Ali et al., 2018), barley stripe mosaic virus (BSMV; Hu et al., 2019), foxtail mosaic virus (FoMV; Mei et al., 2019), beet necrotic yellow vein virus (BNYVV; Jiang et al., 2019), and the single-stranded DNA (ssDNA) cabbage leaf curl virus (CLCV; Yin et al., 2015), were used for delivering sgRNAs and caused intended sequence modification with high efficiency (up to 80\%). Alternatively, virus-mediated genome editing has also been conducted in tobacco (Nicotiana benthamiana) plants using two plant negativestranded RNA (NSR) viruses, barely yellow striate mosaic virus (BYSMV) and sonchus yellow net rhabdovirus (SYNV) that carry Cas9 and sgRNA cassettes simultaneously, and genomeedited lines were generated successfully (Gao et al., 2019; Ma et al., 2020).

Pathways for genome-editing avoiding plant regeneration include transformation of pollen with CRISPR-Cas9 constructs, which has been successfully tested in cotton (Lei et al., 2021), or de novo induction of meristems. De novo induction of meristems has been achieved by using morphogenic regulators (MRs) in plants, and it possesses the potential to produce transgenic plants without the need for a tissue culture procedure (Figure 3C). In general, since plant cells are totipotent and can be trans-differentiated into other cell types, ectopic expression of MRs in somatic cells has the potential to induce meristems. Recently, Agrobacterium harboring sgRNA and $M R$ genes, such as WUSCHEL2 (WUS2), ISOPENTENYL TRANSFERASE (IPT), and SHOOTMERISTEMLESS (STM), was inoculated on the Cas9-overexpressing tobacco ( $N$. benthamiana) plants through pruned sites of plant, and de novo induced meristems from the pruned sites eventually became gene-edited plants (Maher et al., 2020). This delivery system can be applied to various plant species requiring a long tissue culture period in generating transgenic plants.

For the CRISPR-Cas9 transfection to plant cells, two systems - transient or stable expression of CRISPR-Cas9 machinery - can be taken into account based on the time frame and goal of the experiment. In RNP and viral delivery systems, components of the CRISPR-Cas9 system do not get incorporated into the plant genome and the target DNA cleavage engaged by the CRISPR-Cas9 takes place for limited period of time. One of the advantages of transient expression systems is reducing the incidence of off-target events due to the limited editing time. Stable expression of CRISPR-Cas9 agents can be also achieved by using a viral vector, in which Cas9 and/or sgRNA are packaged, resulting in permanent insertion into the cellular genome, and this often involves co-transfecting a selectable antibiotic resistant marker gene for selecting plants with the successful genomic integration. Stable transfections are usually applied for long-term expression of CRISPR-Cas9 agents.

\section{APPLICATION OF GENOME EDITING TO CROP BREEDING}

Genome editing, in particular using CRISPR-Cas9 systems, has been extensively applied to various crops. In most cases, it has been used for complete knockout by specific indel mutations to identify the function of the target genes or produce crops with desired traits (Erpen-Dalla Corte et al., 2019). For example, many genes mainly affecting rice quality, yield, and disease resistance have been modified by CRISPR-Cas9 tools ( $\mathrm{Li}$ et al., 2016b; Lu and Zhu, 2017; Tang et al., 2017; Fiaz et al., 2019). The Waxy gene $(W x)$ encoding a granule-bound starch synthase (GBSS) in rice was modified with the CRISPR-Cas9 system to reduce the amylose content to improve grain quality (Zhang et al., 2018b). Xu et al. (2016) also simultaneously mutated GW2, GW5, and TGW6 that negatively regulate rice grain weight by using the CRISPR-Cas9-mediated multiplex genomeediting system (Xu et al., 2016). The critical residues, NL within the SVLFPNLAGKS sequence of eukaryotic translation initiation factor 4 gamma responsible for the resistance to rice tungro spherical virus, were identified by CRISPR-Cas9-mediated targeted mutagenesis (Macovei et al., 2018). In addition, intron-targeted site-specific gene replacement using CRISPR-Cas9 tools has also been reported in the rice endogenous 5-enolpyruvylshikimate3-phosphate synthase (OsEPSPS); the entire exon 2 of the gene was replaced with a mutated version of the exon containing T102I + P106S amino acid substitutions (Li et al., 2016a). The OsEPSPS mutation caused by the amino acid substitutions conferred resistance to glyphosate.

Maize is one of the most important crops in the world. It is used as a model plant for fundamental genetic research due to its tremendous phenotypic and genotypic diversity (Liu et al., 2020). Feng et al. (2015) introduced a sgRNA-Cas9 construct targeting the $\mathrm{Zmzb}$ that encodes the IspH protein essential for the methyl-D-erythritol-4-phosphate pathway into maize protoplasts (Feng et al., 2015). Indel mutations were detected in regenerated seedlings, and one seedling showed an expected albino phenotype through screening 120 seedlings generated from 10 callus events. Furthermore, CRISPR-Cas9 has also been utilized to create waxy corn hybrids by editing the $W x$ gene in elite inbred maize lines more quickly compared to the conventional trait introgression process. Four CRISPR- $w x$ hybrids displayed a significant increase in grain yield relative to their counterparts (Gao et al., 2020).

In addition, the results of genome editing using the CRISPR-Cas system in tetraploid potato have been released; increased amylopectin/amylose ratio (starch quality) was observed in the line containing all four mutated alleles of the GBSS 
gene with the knockout of GBSS enzyme activity (Andersson et al., 2017). Nakayasu et al. (2018) targeted the St16DOX, encoding a steroid $16 \alpha$-hydroxylase in steroidal glycoalkaloids (SGAs) biosynthesis through CRISPR-Cas9 in order to reduce the level of potato SGAs conferring a bitter taste on human and toxicity against various organisms (Nakayasu et al., 2018). The production of SGA-free hairy roots of tetraploid potato was achieved by using a vector expressing multiplex gRNAs based on the pre-tRNA processing system.

Recently, highly efficient targeted mutagenesis was successfully accomplished by SpRY, an engineered SpCas9 at relaxed PAM sites in a coniferous tree, the Dahurian larch (protoplasts), and in rice transgenic lines (Ren et al., 2021). The case studies clearly demonstrated that the engineered Cas 9 was able to break PAM restriction barriers, allowing researchers to edit anywhere in the genomic sites.

In cases where large deletions yield the desired phenotype, CRISPR-Cas9 mutagenesis targeting multiple sites nearby can be a useful strategy. Four sgRNAs targeting the tomato PMR4 gene, a callose synthase implicated in papillae formation in response to powdery mildew infection over a sequence interval of about 3,700 bp resulted in deletions of up to 900 bp (Santillán Martínez et al., 2020).

Coding sequences were not always targeted by CRISPR-Cas9 mutagenesis. Targeted mutagenesis of promotor sequences was successfully used to alter the expression of target genes ( $\mathrm{Li}$ et al., 2020a). Manipulating gene expression through promotor mutagenesis is particularly useful for genes, where expression changes yield the desired phenotypes and, also for the case that knockouts cause a lethal phenotype, as they have an essential function.

\section{NEW BREEDING STRATEGIES FOR VEGETABLES WITH IMPROVED TRAITS: CASE STUDIES}

Vegetables are recognized as essential crops for the human diet due to their rich nutrient content and phytochemicals that contribute to disease prevention and maintenance of health. Recent research recommended consumption of over 400 grams of vegetables and fruits per day to reduce the risk of being affected by cardiovascular disease or cancer and maintain good health (Aune et al., 2017). Vegetable crops are prone to damage by numerous pests and diseases caused by viruses, bacteria, and fungi, and they are also susceptible to abiotic stresses, such as drought, salinity, flooding, and nutrient deficiency (Hodges and Toivonen, 2008; Boscaiu and Fita, 2020). Therefore, the development of varieties that are resistant to biotic stresses and tolerate abiotic stresses and at the same time have good yields and high nutrient content is the goal of most breeding programs. CRISPR-Cas9 is a candidate technology that can help to reach this goal (WHO, 2003; Karkute et al., 2017; Khatodia et al., 2017).

However, there are many challenges on the way to apply CRISPR-Cas systems in vegetable breeding. First, the genomes of most vegetable species, with the notable exception of tomato, are much less researched than the genomes of staple crops, making the selection of mutagenesis targets more difficult (Li et al., 2019). Accordingly, whole-genome sequence information, well-annotated genes, and functional genomic information are essential to successfully identify the candidate genes for mutagenesis. Second, many traits aimed at by breeders are multigenic, while CRISPR-Cas9 is mostly suitable to target one or a few genetic loci, limiting its application. However, the experiments suggest that site-directed large chromosome rearrangements and translocations are possible (Beying et al., 2020), and these modifications concern many genes, making these approaches more useful to manipulate many linked genes at once. And third, generation of mutants with lossof-function is much easier than those with gain-of-function by CRISPR-Cas9 mutagenesis, representing a further limitation of the technology. Nevertheless, targeting genes with a major function in the trait of interest may result in an improved phenotype.

Based on previously published research articles, information related to the genome editing by CRISPR-Cas systems in vegetables has been collected (Table 1).

\section{Fruit Vegetables \\ Tomato}

Tomato (Solanum lycopersicum L.), owing to the readily available genomic resources and its global economic importance, is the representative vegetable crop where CRISPR-Cas9 approaches have been tested for crop improvement. In this species, the first report on CRISPR-Cas9-mediated genome editing was in 2014, targeting the SIAGAMOUS-LIKE 6 (SIAGL6) gene responsible for leaf development (Brooks et al., 2014). The CRISPR-Cas9 system was also applied to induce targeted mutations in an exon and an untranslated region (UTR) of RIPENING INHIBITOR (RIN) gene, which encodes a MADS-domain transcription factor regulating tomato fruit ripening (Ito et al., 2015). The mutant showed an incomplete ripening phenotype, indicating the importance of functional roles and potential applications of RIN in tomato fruit. Shimatani et al. (2017) utilized a target activation-induced cytidine deaminase (Target-AID; CRISPR-AID) composed of a nCas9 (D10A) and Petromyzon marinus cytidine deaminase 1 (PmCDA1) together with sgRNAs to produce marker-free plants with homozygous heritable DNA substitutions in two endogenous tomato genes, DELLA (Solyc11g011260) or Ethylene Resistance 1 (ETR1; Solyc12g011330) that regulate plant hormone signaling (Arazoe et al., 2017; Shimatani et al., 2017). Moreover, tomato plants with CRISPR-Cas9-mediated slmapk3 (Solanum lycopersicum mitogen-activated protein kinase 3) mutation exhibited enhanced tolerance to heat stress: They showed less severe wilting, milder membrane damage with lower reactive oxygen species (ROS) contents, and presented higher levels of both activity and transcript abundance of antioxidant enzymes under the heat stress condition (Yu et al., 2019). In 2019, Tomlinson et al. reported the first use of CRISPR technology to create a dominant dwarf mutation, $P R O^{\mathrm{D}}$ by modifying the tomato PROCERA gene that encodes a DELLA protein, a key negative regulator of gibberellin 
TABLE 1 | Application of CRISPR-Cas9-based editing of genes in vegetables.

\begin{tabular}{|c|c|c|c|c|c|}
\hline \multicolumn{2}{|c|}{ Vegetable } & \multirow{2}{*}{$\begin{array}{l}\text { Target gene } \\
\text { SIAGO7 }\end{array}$} & \multirow{2}{*}{$\begin{array}{l}\text { Modification/mutant trait } \\
\text { KO/leaflets lacking petioles and later- } \\
\text { formed leaves lacking laminae }\end{array}$} & \multirow{2}{*}{$\begin{array}{l}\text { Delivery method } \\
\text { Agrobacterium-mediated transformation }\end{array}$} & \multirow{2}{*}{$\begin{array}{l}\text { Reference } \\
\text { Brooks et al., } 2014\end{array}$} \\
\hline \multirow{15}{*}{$\begin{array}{l}\text { Fruit } \\
\text { vegetables }\end{array}$} & \multirow{8}{*}{ Tomato } & & & & \\
\hline & & $R / N$ & $\mathrm{KO} /$ incomplete-ripening fruits & Agrobacterium-mediated transformation & Ito et al., 2015 \\
\hline & & DELLA, ETR1 & $\begin{array}{l}\text { Substitutions/marker gene-free plants } \\
\text { harboring stable DNA substitutions }\end{array}$ & Agrobacterium-mediated transformation & Shimatani et al., 2017 \\
\hline & & SLMAPK3 & $\mathrm{KO} /$ reduced drought tolerance & Agrobacterium-mediated transformation & Wang et al., 2017 \\
\hline & & PROCERA & $\mathrm{KO} /$ derepressed growth & Agrobacterium-mediated transformation & Tomlinson et al., 2019 \\
\hline & & SICLV3 & $\begin{array}{l}\text { Mutation in SICLV3 promoter/ } \\
\text { phenotypic changes in fruit size, } \\
\text { flower morphology, and locule number }\end{array}$ & Agrobacterium-mediated transformation & $\begin{array}{l}\text { Rodríguez-Leal et al., } \\
2017\end{array}$ \\
\hline & & $\begin{array}{l}\text { SP, SP5G, SICLV3, } \\
\text { SIWUS }\end{array}$ & $\begin{array}{l}\text { Gene editing of coding sequences, cis- } \\
\text { regulatory regions, or upstream open } \\
\text { reading frames (ORF)/de novo- } \\
\text { domesticated tomato }\end{array}$ & Agrobacterium-mediated transformation & Li et al., 2018b \\
\hline & & $\begin{array}{l}S P, O, F W 2.2 \\
\text { CycB, FAS, MULT }\end{array}$ & $\begin{array}{l}\text { Simultaneous CRISPR-Cas9 editing of } \\
\text { six genes/modification of fruit number, } \\
\text { size, shape, nutrient content, and plant } \\
\text { architecture }\end{array}$ & Agrobacterium-mediated transformation & Zsögön et al., 2018 \\
\hline & \multirow[t]{2}{*}{ Cucumber } & elF4E & $\begin{array}{l}\mathrm{KO} / \text { resistance to ipomovirus, } \\
\text { potyviruses zucchini yellow mosaic } \\
\text { virus and papaya ring spot mosaic } \\
\text { virus-W }\end{array}$ & Agrobacterium-mediated transformation & $\begin{array}{l}\text { Chandrasekaran et al., } \\
2016\end{array}$ \\
\hline & & CsWIP1 & KO/gynoecious phenotype & $\begin{array}{l}\text { Enhanced Agrobacterium-mediated } \\
\text { transformation using vacuum infiltration }\end{array}$ & Hu et al., 2017 \\
\hline & \multirow{4}{*}{ Watermelon } & CIPDS & KO/albino phenotype & $\begin{array}{l}\text { Agrobacterium-mediated transformation / } \\
\text { PEG-mediated protoplast transfection }\end{array}$ & Tian et al., 2017 \\
\hline & & CIALS & Point mutation/herbicide resistance & Agrobacterium-mediated transformation & Tian et al., 2018 \\
\hline & & CIPSK1 & $\begin{array}{l}\text { KO/enhanced resistance to Fusarium } \\
\text { oxysporum f. sp. niveum }\end{array}$ & Agrobacterium-mediated transformation & Zhang et al., 2020b \\
\hline & & CIWIP1 & KO/gynoecious watermelon & Agrobacterium-mediated transformation & Zhang et al., 2020a \\
\hline & Eggplant & SmelPPO & $\begin{array}{l}\mathrm{KO} / \text { lowered enzymatic browning in } \\
\text { eggplant berries }\end{array}$ & Agrobacterium-mediated transformation & Maioli et al., 2020 \\
\hline \multirow{9}{*}{$\begin{array}{l}\text { Leafy } \\
\text { vegetables }\end{array}$} & \multirow{3}{*}{ Lettuce } & LsBIN2 & $\begin{array}{l}\mathrm{KO} / \text { targeted gene disruption in whole } \\
\text { plants regenerated from protoplasts }\end{array}$ & PEG-mediated protoplast transfection & Woo et al., 2015 \\
\hline & & LsNCED4 & $\mathrm{KO} /$ loss of thermoinhibition & $\begin{array}{l}\text { Agrobacterium-mediated callus or } \\
\text { somatic explants transformation }\end{array}$ & Bertier et al., 2018 \\
\hline & & LsGGP2 & $\begin{array}{l}\text { Deleted uORFs of LsGGP2 for } \\
\text { increasing the translation of mRNAs/ } \\
\text { increased oxidation stress tolerance } \\
\text { and ascorbate content }\end{array}$ & $\begin{array}{l}\text { Agrobacterium-mediated callus or } \\
\text { somatic explants transformation }\end{array}$ & Zhang et al., 2018a \\
\hline & Chicory & CiPDS & KO/albino phenotype & $\begin{array}{l}\text { Agrobacterium-mediated transformation/ } \\
\text { PEG-mediated protoplast transfection }\end{array}$ & Bernard et al., 2019 \\
\hline & \multirow[b]{2}{*}{ Chinese kale } & $B a P D S 1, B a P D S 2$ & $\mathrm{KO}$ or KD/albino phenotype & Agrobacterium-mediated transformation & Sun et al., 2018 \\
\hline & & BoaCRTISO & $\begin{array}{l}\text { KD/yellow color of Chinese kale with } \\
\text { improved market prospects }\end{array}$ & Agrobacterium-mediated transformation & Sun et al., 2020 \\
\hline & \multirow[b]{2}{*}{ Cabbage } & BoPDS & KO/albino phenotype & $\begin{array}{l}\text { Agrobacterium-mediated transformation } \\
\text { Electro-transfection in RNP delivery to } \\
\text { protoplast }\end{array}$ & $\begin{array}{l}\text { Ma et al., 2019a } \\
\text { Lee et al., } 2020\end{array}$ \\
\hline & & $\begin{array}{l}\text { BoPDS1, BoSRK3, } \\
\text { BoMS1 }\end{array}$ & $\begin{array}{l}\text { Multisite and multiple gene } \mathrm{KO} \text { using } \\
\text { an array of sgRNA-tRNA/male-sterile } \\
\text { line }\end{array}$ & $\begin{array}{l}\text { Agrobacterium-mediated hypocotyls } \\
\text { transformation }\end{array}$ & Ma et al., 2019b \\
\hline & $\begin{array}{l}\text { Chinese } \\
\text { cabbage }\end{array}$ & BraFLCs & $\begin{array}{l}\text { KO/early-flowering phenotype that did } \\
\text { not depend on vernalization }\end{array}$ & Agrobacterium-mediated transformation & Jeong et al., 2019 \\
\hline
\end{tabular}

signaling (Yoshida et al., 2014). They analyzed phenotypes of heterozygous and homozygous mutant plants for $P R O^{\mathrm{D}}: P R O^{\mathrm{D}} / P R O^{\mathrm{D}}$ and $P R O^{\mathrm{D}} / P R O$. At the seedling stage, $P R O^{\mathrm{D}} / P R O$ plants exhibited an intermediate phenotype in plant height between WT $(P R O / P R O)$ and homozygous $\left(P R O^{\mathrm{D}} / P R O^{\mathrm{D}}\right)$ plants indicating that the mutation is semidominant at that stage. Later in development, however, both heterozygous and homozygous plants for $P R O^{\mathrm{D}}$ were equally dwarfed compared to WT (Tomlinson et al., 2019). Compared to mutations in the coding region to create null alleles, the advent of CRISPR-Cas9-mediated engineering in cis-regulatory elements (CREs) required for the expressional 
regulation of a coding sequence provides a more refined method for creating phenotypic diversity for enhancing crop breeding (Swinnen et al., 2016; Wolter and Puchta, 2018). Indeed, multiplexed CRISPR-Cas9 mutagenesis in the SlCLV3 promoter region created a series of cis-regulatory alleles, resulting in a range of changes from the transcriptional level of SlCLV3 to phenotypes in fruit size, flower morphology, and locule number. Hence, this approach offers the possibility of more efficient breeding by fine-tuning the expression of genes associated with improved yield and crop quality (Rodríguez-Leal et al., 2017).

The capacity to simultaneously target multiple genes for editing facilitated an experiment to perform some major steps in "redomestication" of tomato. Through CRISPR-Cas9-mediated disruption of six independent genes in wild tomato (Solanum pimpinellifolium), fruit size and fruit number in mutated wild tomato were increased three- and ten-fold, respectively (Zsögön et al., 2018). Of note, the "redomesticated" tomato with improved fruit and agronomic traits retained the increased tolerance to salt stress and disease (Li et al., 2018b). Besides these approaches aiming at phenotypic changes, CRISPR-Cas9 became a routine technology for functional genomics, including validation of candidate genes identified in genome-wide association studies (Alonge et al., 2020).

\section{Cucumber}

Cucumber (Cucumis sativus L.), belonging to the Cucurbitaceae family, is also an economically important vegetable (Huang et al., 2009) that is cultivated in nearly all countries within both temperate and tropical zones (Tatlioglu, 1993). The first application of CRISPR-Cas9 in cucumber was for conferring broad viral resistance through knockout of eukaryotic translation initiation factor 4E (eIF4E) gene (Chandrasekaran et al., 2016). Gynoecious inbred lines in cucumber have great importance due to their higher production yield and the lower labor cost required for crossing (Robinson, 2000). Hu et al. (2017) generated Cswip1 mutants by CRISPR-Cas9 tools, targeting the WPP trp/ pro/pro domain Interacting Protein1 (CsWIP1) gene, which encodes a zinc-finger transcription factor. Cswip1 T0 mutants exhibited gynoecious phenotype bearing only female flowers, implying that the gene is involved in inhibition of cucumber carpel development (Hu et al., 2017).

\section{Watermelon}

Watermelon (Citrullus lanatus), which belongs to the Cucurbitaceae family, is a rich source of citrulline, vitamins, and lycopene (Collins et al., 2007; Maoto et al., 2019). CRISPR-Cas9-mediated mutations in the phytoene desaturase (ClPDS) gene, encoding a key enzyme of carotenoid synthesis, caused the expected albino phenotype in watermelon plants (Tian et al., 2017). The CRISPR-Cas9-mediated base editing system was also utilized to achieve single-nucleotide conversion at the acetolactate synthase (ClALS) gene in watermelon (Tian et al., 2018). Watermelon plants possessing $\mathrm{C}$ to $\mathrm{T}$ mutations in the codon of Pro 190 (CCG) at the ClALS gene have become resistant to all sulfonylurea herbicides without compromising fruit and seed size, and seed yield (Yu and Powles, 2014). In addition, the CRISPR-Cas9 system was used to generate the knockout mutation of the phytosulfokine1 (ClPSK1) gene responsible for the infection by Fusarium oxysporum $\mathrm{f}$. sp. niveum (FON). The loss-of-function mutation of ClPSK1 rendered watermelon seedlings more resistant to infection by FON (Zhang et al., 2020b). Recently, it was reported that the ClWIP1, a homologue of CsWIP1 and CmWIP1 in cucumber and melon, respectively, acts as the gynoecious (gy) gene in watermelon (Martin et al., 2009). The ClWIP1 is specifically expressed in carpel primordia in male floral buds and also linked to the abortion of carpel primordia in early floral development. Artificial gynoecious watermelon lines have been generated using the CRISPR-Cas9 system targeting the ClWIP1 (Zhang et al., 2020a).

\section{Eggplant}

Eggplant (Solanum melongena L.) ranks fifth among vegetables in terms of total global production, with 52.3 million tons produced in 2017 (Alam and Salimullah, 2021). Three polyphenol oxidase genes (PPOs; SmelPPO4, SmelPPO5, and SmelPPO6) showing the highest transcript levels in the fruit after cutting were regarded to be associated with enzymatic browning of eggplants, and CRISPR-Cas9-based mutagenesis has been applied to knockout three target $P P O$ genes simultaneously aiming to reduce fruit flesh browning (Maioli et al., 2020).

\section{Leafy Vegetables \\ Lettuce}

Leafy vegetables have also been edited for trait improvement through CRISPR-Cas applications. In particular, a DNA-free genome-editing approach has been applied in lettuce and cabbage via Cas9- and Cpf1-RNPs delivery into protoplasts. Woo et al. (2015) succeeded in delivering CRISPR-Cas9 RNPs into lettuce protoplasts by PEG-mediated transfection and, subsequently, regenerated plants with intended mutations from the protoplasts (Woo et al., 2015). Recently, it has been suggested that electroporation is more efficient in RNP delivery to protoplasts than PEG-mediated transfection in cabbage based on the results of phytoene desaturase1 (PDS1) sgRNA delivery, which may be due to lower chemical toxicity (Lee et al., 2020). It has also been found that genome editing of upstream open reading frame (uORF) enabled the modulation of translation of mRNA. Editing the uORF of LsGGP1 and LsGGP2, which encodes a key enzyme in vitamin C biosynthesis, increased mRNA translation, thereby elevating ascorbate content and oxidation stress tolerance.

\section{Other Leafy Vegetables}

Like other vegetables, the CRISPR-Cas9-based gene knockout or knockdown was applied to PDS genes in chicory (Cichorium intybus L.; Bernard et al., 2019), Chinese kale (Brassica oleracea var. alboglabra), and cabbage (Brassica oleracea var. capitata; Ma et al., 2019a,b; Lee et al., 2020), and PDS mutants were detected by their albino phenotype. CRISPR-Cas9 was also used to induce mutations in the carotenoid isomerase gene (BoaCRTISO) of Chinese kale (Sun et al., 2020). The color of 
biallelic and homozygous CRTISO mutants was changed from green to yellow in the leaves and bolting stems. Also, Jeong et al. (2019) succeeded in generating the early flowering Chinese cabbage (Brassica rapa spp. pekinensis) by the CRISPR-Cas9mediated knockout in FLOWERING LOCUS C (BraFLC) genes homologous to Arabidopsis FLC, which encodes a MADS domain protein that plays a central role in repressing flowering (Deng et al., 2011; Jeong et al., 2019).

\section{PERSPECTIVE}

The CRISPR-Cas systems confer significant opportunities for improvement in crop production by mitigating biotic and abiotic stresses as well as increasing yield (Doudna and Charpentier, 2014; Langner et al., 2018). Many consumers believe that the production of genetically modified organisms (GMOs) is risky for the environment and health and that it may be dangerous to consume GMOs mostly due to lack of consumer knowledge about GMOs (Wunderlich and Gatto, 2015). However, gene editing using CRISPR-Cas9 systems differs noticeably from the conventional genetic modification in the 1990s. While conventional genetic modification introduces foreign DNA into a crop, CRISPR, like mutation breeding, only induces small changes in the native DNA, and in contrast to mutation breeding, these changes are not random but generally well defined (Shew et al., 2018). But in any case, plants released into the environment must be transgene free as CRISPR geneediting constructs are potentially hazardous to the environment (Huang et al., 2016). Furthermore, traits in commercial crops must be stable, and a functioning CRISPR-Cas9 system in plants may cause phenotypic variation through off-target mutations (He and Zhao, 2020). In sexually propagated plants, the CRISPR-Cas 9 cassette could be eliminated by crosses and marker-assisted selection of segregants carrying the mutation but not the CRISPR-Cas9 cassette, or transgene-free cells can be selected expressing suicide genes in a temporarycontrolled manner.

Off-target mutagenesis through CRISPR-Cas9 is considered a risk for medical approaches in humans and animals, but much less so in plants, where suitable phenotypes can be selected from a large number of mutant genotypes. Moreover, recent results clearly indicate that off-target effects through genome editing occur by orders of magnitude less frequently, compared to chemical or irradiation methods to induce mutations in plants (Modrzejewski et al., 2020).

Nevertheless, analyses methods for nontarget mutations are becoming more efficient (Liu et al., 2021a), and tools available for animal research could also be adapted for plants to assess the unexpected effects of genome editing (Liu et al., 2021b). Generally, one of the two global regulatory policies for genome-editing system has been used in different countries (i.e., product based and process based; Globus and Qimron, 2018). The United States (Kleter et al., 2019), Argentina (Gao et al., 2018), and many other countries have adopted a product-based approach, which evaluates the safety of genome-modified end-products, while the European Union
(Jouanin et al., 2018) and New Zealand (Fritsche et al., 2018) have chosen to regulate genome-edited products based on the process used. And if recombinant DNA technology is used to produce the mutated plant, according to a processbased regulation, it is considered to be a genetically modified organism. According to these regulatory policies, genomeedited plants through CRISPR-Cas9 systems have been differently classified as GMOs or non-GMOs in different countries. CRISPR-Cas9 regulation is not finalized in most countries; some countries classified edited plants without a transgene [the so-called site directed nuclease technology-1 (SDN-1) events] as non-GMO, while other countries consider edited plants as GMO (Zhang et al., 2020c). SDN-2 events, like SDN1 events, are produced with a DSB, but for repair of the break, a small nucleotide template complementary to the area of the break is provided to cause the insertion of a short sequence into the gap. SDN-2 events, in the strict sense, do not contain foreign DNA (except a few additional bases) and therefore are not considered as GMO in most countries with a product-based gene-editing regulation. SDN-3 events are analogously produced, like SDN-2 events, but the template provided to guide the repair contains a gene or other sequence. SDN-3 plants therefore are generally considered as GMO.

There is currently ongoing controversy about the regulation of new gene-editing techniques because the strict regulatory policies, especially the regulatory framework in the EU, will restrict the application of genome-editing technology which has tremendous potential for improving crops although a very recent report from the European Commission presented a positive viewpoint on innovation in gene editing with maintaining a cautionary tone (Friedrich, 2020). ${ }^{1}$ More efficient and robust gene-/genome-editing systems, including the components of their delivery systems with fewer off-target effects for plant species, will be updated continuously. Particularly, multiple omics approaches, such as whole genome sequencing, RNA/ small RNA-seq, and proteome analyses, will be of great help in finding more target genes for gene-/genome-editing-based vegetable improvement because selecting/finding appropriate genes for a desired trait is the key for the ultimate generation of improved vegetables through precise manipulation of genetic elements required for phenotypic alterations, particularly for the traits with a complicated genetic background, such as yield and quality. Thus, continuous efforts to identify genetic players that directly or indirectly control desirable traits and serve as targets for genome editing in vegetables are also required.

\section{AUTHOR CONTRIBUTIONS}

All authors listed have made a substantial and intellectual contribution to the work and approved the final version of the manuscript for publication.

${ }^{1}$ https://ec.europa.eu/food/sites/food/files/plant/docs/gmo_mod-bio_ngt_ eu-study.pdf 


\section{FUNDING}

This work was supported in part by grants (Project No. PJ01480101 to E-YY and Project No. PJ01532503 to JL) from the Rural Development Administration (RDA), Republic of Korea, and a grant from the World Vegetable Center Korea

\section{REFERENCES}

Abdallah, N. A., Prakash, C. S., and McHughen, A. G. (2015). Genome editing for crop improvement: challenges and opportunities. GM Crops Food 6, 183-205. doi: 10.1080/21645698.2015.1129937

Ahloowalia, B. S., Maluszynski, M., and Nichterlein, K. (2004). Global impact of mutation-derived varieties. Euphytica 135, 187-204. doi: 10.1023/ B:EUPH.0000014914.85465.4f

Ainley, W. M., Sastry-Dent, L., Welter, M. E., Murray, M. G., Zeitler, B., Amora, R., et al. (2013). Trait stacking via targeted genome editing. Plant Biotechnol. J. 11, 1126-1134. doi: 10.1111/pbi.12107

Alam, I., and Salimullah, M. (2021). Genetic engineering of eggplant (Solanum melongena L.): progress, controversy and potential. Horticulturae 7:78. doi: 10.3390/horticulturae7040078

Ali, Z., Abul-faraj, A., Li, L., Ghosh, N., Piatek, M., Mahjoub, A., et al. (2015). Efficient virus-mediated genome editing in plants using the CRISPR/Cas9 system. Mol. Plant 8, 1288-1291. doi: 10.1016/j.molp.2015.02.011

Ali, Z., Eid, A., Ali, S., and Mahfouz, M. M. (2018). Pea early-browning virusmediated genome editing via the CRISPR/Cas9 system in Nicotiana benthamiana and Arabidopsis. Virus Res. 244, 333-337. doi: 10.1016/j.virusres.2017.10.009

Alkan, F., Wenzel, A., Anthon, C., Havgaard, J. H., and Gorodkin, J. (2018). CRISPR-Cas9 off-targeting assessment with nucleic acid duplex energy parameters. Genome Biol. 19:177. doi: 10.1186/s13059-018-1534-x

Alonge, M., Wang, X., Benoit, M., Soyk, S., Pereira, L., Zhang, L., et al. (2020). Major impacts of widespread structural variation on gene expression and crop improvement in tomato. Cell 182, 145.e23-161.e23. doi: 10.1016/j. cell.2020.05.021

Andersson, M., Turesson, H., Nicolia, A., Fält, A.-S., Samuelsson, M., and Hofvander, P. (2017). Efficient targeted multiallelic mutagenesis in tetraploid potato (Solanum tuberosum) by transient CRISPR-Cas9 expression in protoplasts. Plant Cell Rep. 36, 117-128. doi: 10.1007/s00299-016-2062-3

Anzalone, A. V., Randolph, P. B., Davis, J. R., Sousa, A. A., Koblan, L. W., Levy, J. M., et al. (2019). Search-and-replace genome editing without doublestrand breaks or donor DNA. Nature 576, 149-157. doi: 10.1038/ s41586-019-1711-4

Arazoe, T., Nishida, K., and Kondo, A. (2017). Targeted nucleotide substitution in mammalian cell by target-AID. Bio-Protocol 7:e2339. doi: 10.21769/ BioProtoc. 2339

Arora, L., and Narula, A. (2017). Gene editing and crop improvement using CRISPR-Cas9 system. Front. Plant Sci. 8:1932. doi: 10.3389/fpls.2017.01932

Atkins, P. A., and Voytas, D. F. (2020). Overcoming bottlenecks in plant gene editing. Curr. Opin. Plant Biol. 54, 79-84. doi: 10.1016/j.pbi.2020.01.002

Aune, D., Giovannucci, E., Boffetta, P., Fadnes, L. T., Keum, N., Norat, T., et al. (2017). Fruit and vegetable intake and the risk of cardiovascular disease, total cancer and all-cause mortality-a systematic review and doseresponse meta-analysis of prospective studies. Int. J. Epidemiol. 46, 1029-1056. doi: 10.1093/ije/dyw319

Barrangou, R. (2013). CRISPR-Cas systems and RNA-guided interference. Wiley Interdiscip. Rev. RNA 4, 267-278. doi: 10.1002/wrna.1159

Bernard, G., Gagneul, D., Alves Dos Santos, H., Etienne, A., Hilbert, J.-L., and Rambaud, C. (2019). Efficient genome editing using CRISPR/Cas9 technology in chicory. Int. J. Mol. Sci. 20:1155. doi: 10.3390/ijms20051155

Bernardo, R. (2016). Bandwagons I, too, have known. Theor. Appl. Genet. 129, 2323-2332. doi: 10.1007/s00122-016-2772-5

Bertier, L. D., Ron, M., Huo, H., Bradford, K. J., Britt, A. B., and Michelmore, R. W. (2018). High-resolution analysis of the efficiency, heritability, and editing outcomes of CRISPR/Cas9-induced modifications of NCED4 in lettuce (Lactuca sativa). G3: Genes Genom. Genet. 8, 1513-1521. doi: 10.1534/ g3.117.300396
Office (WKO \#10000379), core donors to the World Vegetable Center: Republic of China (ROC), UK Aid from the UK Government, the United States Agency for International Development (USAID), and the Australian Center for International Agricultural Research (ACIAR), Germany, Thailand, Philippines, Korea, and Japan.

Beying, N., Schmidt, C., Pacher, M., Houben, A., and Puchta, H. (2020). CRISPR-Cas9-mediated induction of heritable chromosomal translocations in Arabidopsis. Nat. Plants 6, 638-645. doi: 10.1038/s41477-020-0663-X

Bhakta, M. S., Henry, I. M., Ousterout, D. G., Das, K. T., Lockwood, S. H., Meckler, J. F., et al. (2013). Highly active zinc-finger nucleases by extended modular assembly. Genome Res. 23, 530-538. doi: 10.1101/gr. 143693.112

Bhowmik, P., Ellison, E., Polley, B., Bollina, V., Kulkarni, M., Ghanbarnia, K., et al. (2018). Targeted mutagenesis in wheat microspores using CRISPR/ Cas9. Sci. Rep. 8:6502. doi: 10.1038/s41598-018-24690-8

Bisbis, M. B., Gruda, N., and Blanke, M. (2018). Potential impacts of climate change on vegetable production and product quality - a review. J. Clean. Prod. 170:1602. doi: 10.1016/j.jclepro.2017.09.224

Bitinaite, J., Wah, D. A., Aggarwal, A. K., and Schildkraut, I. (1998). FokI dimerization is required for DNA cleavage. Proc. Natl. Acad. Sci. U. S. A. 95, 10570-10575. doi: 10.1073/pnas.95.18.10570

Boch, J., and Bonas, U. (2010). Xanthomonas AvrBs3 family-type III effectors: discovery and function. Annu. Rev. Phytopathol. 48, 419-436. doi: 10.1146/ annurev-phyto-080508-081936

Borrelli, V. M. G., Brambilla, V., Rogowsky, P., Marocco, A., and Lanubile, A. (2018). The enhancement of plant disease resistance using CRISPR/Cas9 technology. Front. Plant Sci. 9:1245. doi: 10.3389/fpls.2018.01245

Boscaiu, M., and Fita, A. (2020). Physiological and molecular characterization of crop resistance to abiotic stresses. Agronomy 10:1308. doi: 10.3390/ agronomy 10091308

Brandt, K., and Barrangou, R. (2019). Applications of CRISPR technologies across the food supply chain. Annu. Rev. Food Sci. Technol. 10, 133-150. doi: 10.1146/annurev-food-032818-121204

Brooks, C., Nekrasov, V., Lippman, Z. B., and Van Eck, J. (2014). Efficient gene editing in tomato in the first generation using the clustered regularly interspaced short palindromic repeats/CRISPR-associated9 system. Plant Physiol. 166, 1292-1297. doi: 10.1104/pp.114.247577

Burstein, D., Harrington, L. B., Strutt, S. C., Probst, A. J., Anantharaman, K., Thomas, B. C., et al. (2017). New CRISPR-Cas systems from uncultivated microbes. Nature 542, 237-241. doi: 10.1038/nature21059

Cantos, C., Francisco, P., Trijatmiko, K. R., Slamet-Loedin, I., and Chadha-Mohanty, P. K. (2014). Identification of "safe harbor" loci in indica rice genome by harnessing the property of zinc-finger nucleases to induce DNA damage and repair. Front. Plant Sci. 5:302. doi: 10.3389/fpls.2014. 00302

Chandrasekaran, J., Brumin, M., Wolf, D., Leibman, D., Klap, C., Pearlsman, M., et al. (2016). Development of broad virus resistance in non-transgenic cucumber using CRISPR/Cas9 technology. Mol. Plant Pathol. 17, 1140-1153. doi: $10.1111 / \mathrm{mpp} .12375$

Chaudhary, J., Alisha, A., Bhatt, V., Chandanshive, S., Kumar, N., Mir, Z., et al. (2019). Mutation breeding in tomato: advances, applicability and challenges. Plan. Theory 8:128. doi: 10.3390/plants8050128

Chen, K., and Gao, C. (2014). Targeted genome modification technologies and their applications in crop improvements. Plant Cell Rep. 33, 575-583. doi: 10.1007/s00299-013-1539-6

Chen, K., Wang, Y., Zhang, R., Zhang, H., and Gao, C. (2019). CRISPR/Cas genome editing and precision plant breeding in agriculture. Annu. Rev. Plant Biol. 70, 667-697. doi: 10.1146/annurev-arplant-050718-100049

Christian, M., Cermak, T., Doyle, E. L., Schmidt, C., Zhang, F., Hummel, A., et al. (2010). Targeting DNA double-strand breaks with TAL effector nucleases. Genetics 186, 757-761. doi: 10.1534/genetics.110.120717

Cody, W. B., Scholthof, H. B., and Mirkov, T. E. (2017). Multiplexed gene editing and protein overexpression using a tobacco mosaic virus viral vector. Plant Physiol. 175, 23-35. doi: 10.1104/pp.17.00411 
Collins, J. K., Wu, G., Perkins-Veazie, P., Spears, K., Claypool, P. L., Baker, R. A., et al. (2007). Watermelon consumption increases plasma arginine concentrations in adults. Nutrition 23, 261-266. doi: 10.1016/j.nut.2007.01.005

Cong, L., Ran, F. A., Cox, D., Lin, S., Barretto, R., Habib, N., et al. (2013). Multiplex genome engineering using CRISPR/Cas systems. Science 339, 819-823. doi: 10.1126/science.1231143

Curtin, S. J., Zhang, F., Sander, J. D., Haun, W. J., Starker, C., Baltes, N. J., et al. (2011). Targeted mutagenesis of duplicated genes in soybean with zinc-finger nucleases. Plant Physiol. 156, 466-473. doi: 10.1104/pp.111.172981

Deng, W., Ying, H., Helliwell, C. A., Taylor, J. M., Peacock, W. J., and Dennis, E. S. (2011). FLOWERING LOCUS C (FLC) regulates development pathways throughout the life cycle of Arabidopsis. Proc. Natl. Acad. Sci. U. S. A. 108, 6680-6685. doi: 10.1073/pnas.1103175108

Doudna, J. A., and Charpentier, E. (2014). The new frontier of genome engineering with CRISPR-Cas9. Science 346:1258096. doi: 10.1126/science.1258096

Eid, A., and Mahfouz, M. (2016). Genome editing: the road of CRISPR/Cas9 from bench to clinic. Exp. Mol. Med. 48:e265. doi: 10.1038/emm.2016.111

Erpen-Dalla Corte, L., M Mahmoud, L., S Moraes, T., Mou, Z., W Grosser, J., and Dutt, M. (2019). Development of improved fruit, vegetable, and ornamental crops using the CRISPR/Cas9 genome editing technique. Plants 8:601. doi: $10.3390 /$ plants 8120601

Esvelt, K. M., Mali, P., Braff, J. L., Moosburner, M., Yaung, S. J., and Church, G. M. (2013). Orthogonal Cas9 proteins for RNA-guided gene regulation and editing. Nat. Methods 10, 1116-1121. doi: 10.1038/nmeth.2681

Feng, C., Yuan, J., Wang, R., Liu, Y., Birchler, J., and Han, F. (2015). Efficient targeted genome modification in maize using CRISPR/Cas9 System. J. Genet. Genomics 43, 37-43. doi: 10.1016/j.jgg.2015.10.002

Fiaz, S., Ahmad, S., Noor, M. A., Wang, X., Younas, A., Riaz, A., et al. (2019). Applications of the CRISPR/Cas9 system for rice grain quality improvement: perspectives and opportunities. Int. J. Mol. Sci. 20:888. doi: 10.3390/ ijms 20040888

Filler Hayut, S., Melamed Bessudo, C., and Levy, A. A. (2017). Targeted recombination between homologous chromosomes for precise breeding in tomato. Nat. Commun. 8:15605. doi: 10.1038/ncomms15605

Fischer, S., Maier, L. K., Stoll, B., Brendel, J., Fischer, E., Pfeiffer, F., et al. (2012). An archaeal immune system can detect multiple protospacer adjacent motifs (PAMs) to target invader DNA. J. Biol. Chem. 287, 33351-33363. doi: $10.1074 /$ jbc.M112.377002

Friedrich, B. (2020). Pathways of conflict: lessons from the cultivation of MON810 in Germany in 2005-2008 for emerging conflicts over new breeding techniques. Sustainability 12:144. doi: 10.3390/su12010144

Fritsche, S., Poovaiah, C., MacRae, E., and Thorlby, G. (2018). A new zealand perspective on the application and regulation of gene editing. Front. Plant Sci. 9:1323. doi: $10.3389 /$ fpls.2018.01323

Gaj, T., Gersbach, C. A., and Barbas, C. F. 3rd. (2013). ZFN, TALEN, and CRISPR/Cas-based methods for genome engineering. Trends Biotechnol. 31, 397-405. doi: 10.1016/j.tibtech.2013.04.004

Gao, C. (2014). Genome editing in crops: from bench to field. Natl. Sci. Rev. 2, 13-15. doi: 10.1093/nsr/nwu054

Gao, H., Gadlage, M. J., Lafitte, H. R., Lenderts, B., Yang, M., Schroder, M., et al. (2020). Superior field performance of waxy corn engineered using CRISPR-Cas9. Nat. Biotechnol. 38, 579-581. doi: 10.1038/s41587-020-0444-0

Gao, J., Wang, G., Ma, S., Xie, X., Wu, X., Zhang, X., et al. (2015). CRISPR/ Cas9-mediated targeted mutagenesis in Nicotiana tabacum. Plant Mol. Biol. 87, 99-110. doi: 10.1007/s11103-014-0263-0

Gao, L., Cox, D. B. T., Yan, W. X., Manteiga, J. C., Schneider, M. W., Yamano, T., et al. (2017). Engineered Cpfl variants with altered PAM specificities. Nat. Biotechnol. 35, 789-792. doi: 10.1038/nbt.3900

Gao, Q., Xu, W. Y., Yan, T., Fang, X. D., Cao, Q., Zhang, Z. J., et al. (2019). Rescue of a plant cytorhabdovirus as versatile expression platforms for planthopper and cereal genomic studies. New Phytol. 223, 2120-2133. doi: 10.1111/nph.15889

Gao, W., Xu, W.-T., Huang, K.-L., Guo, M.-Z., and Luo, Y.-B. (2018). Risk analysis for genome editing-derived food safety in China. Food Control 84, 128-137. doi: 10.1016/j.foodcont.2017.07.032

Gasiunas, G., Barrangou, R., Horvath, P., and Siksnys, V. (2012). Cas9-crRNA ribonucleoprotein complex mediates specific DNA cleavage for adaptive immunity in bacteria. Proc. Natl. Acad. Sci. 109, E2579-E2586. doi: 10.1073/ pnas. 1208507109
Gaudelli, N. M., Komor, A. C., Rees, H. A., Packer, M. S., Badran, A. H., Bryson, D. I., et al. (2017). Programmable base editing of A•T to G.C in genomic DNA without DNA cleavage. Nature 551, 464-471. doi: 10.1038/ nature24644

Gaudelli, N. M., Lam, D. K., Rees, H. A., Solá-Esteves, N. M., Barrera, L. A., Born, D. A., et al. (2020). Directed evolution of adenine base editors with increased activity and therapeutic application. Nat. Biotechnol. 38, 892-900. doi: 10.1038/s41587-020-0491-6

Giraut, L., Falque, M., Drouaud, J., Pereira, L., Martin, O. C., and Mézard, C. (2011). Genome-wide crossover distribution in Arabidopsis thaliana meiosis reveals sex-specific patterns along chromosomes. PLoS Genet. 7:e1002354. doi: 10.1371/journal.pgen.1002354

Glenn, K., Alsop, B., Bell, E., Goley, M., Jenkinson, J., Liu, B., et al. (2017). Bringing new plant varieties to market: plant breeding and selection practices advance beneficial characteristics while minimizing unintended changes. Crop Sci. 57, 2906-2921. doi: 10.2135/cropsci2017.03.0199

Globus, R., and Qimron, U. (2018). A technological and regulatory outlook on CRISPR crop editing. J. Cell. Biochem. 119, 1291-1298. doi: 10.1002/ jcb. 26303

Gupta, R. M., and Musunuru, K. (2014). Expanding the genetic editing tool kit: ZFNs, TALENs, and CRISPR-Cas9. J. Clin. Invest. 124, 4154-4161. doi: $10.1172 / J C I 72992$

Haun, W., Coffman, A., Clasen, B. M., Demorest, Z. L., Lowy, A., Ray, E., et al. (2014). Improved soybean oil quality by targeted mutagenesis of the fatty acid desaturase 2 gene family. Plant Biotechnol. J. 12, 934-940. doi: $10.1111 /$ pbi.12201

He, Y., and Zhao, Y. (2020). Technological breakthroughs in generating transgenefree and genetically stable CRISPR-edited plants. aBIOTECH 1, 88-96. doi: 10.1007/s42994-019-00013-x

Hodges, D. M., and Toivonen, P. M. A. (2008). Quality of fresh-cut fruits and vegetables as affected by exposure to abiotic stress. Postharvest Biol. Technol. 48, 155-162. doi: 10.1016/j.postharvbio.2007.10.016

Holme, I. B., Gregersen, P. L., and Brinch-Pedersen, H. (2019). Induced genetic variation in crop plants by random or targeted mutagenesis: convergence and differences. Front. Plant Sci. 10:1468. doi: 10.3389/fpls.2019.01468

Horvath, P., and Barrangou, R. (2010). CRISPR/Cas, the immune system of bacteria and archaea. Science 327, 167-170. doi: 10.1126/science. 1179555

Hu, B., Li, D., Liu, X., Qi, J., Gao, D., Zhao, S., et al. (2017). Engineering non-transgenic gynoecious cucumber using an improved transformation protocol and optimized CRISPR/Cas9 system. Mol. Plant 10, 1575-1578. doi: 10.1016/j.molp.2017.09.005

Hu, J., Li, S., Li, Z., Li, H., Song, W., Zhao, H., et al. (2019). A barley stripe mosaic virus-based guide RNA delivery system for targeted mutagenesis in wheat and maize. Mol. Plant Pathol. 20, 1463-1474. doi: 10.1111/mpp. 12849

Hu, J. H., Miller, S. M., Geurts, M. H., Tang, W., Chen, L., Sun, N., et al. (2018). Evolved Cas9 variants with broad PAM compatibility and high DNA specificity. Nature 556, 57-63. doi: 10.1038/nature26155

Hua, K., Tao, X., and Zhu, J. K. (2019). Expanding the base editing scope in rice by using Cas9 variants. Plant Biotechnol. J. 17, 499-504. doi: 10.1111/ pbi. 12993

Huang, S., Li, R., Zhang, Z., Li, L., Gu, X., Fan, W., et al. (2009). The genome of the cucumber, Cucumis sativus L. Nat. Genet. 41, 1275-1281. doi: 10.1038/ ng. 475

Huang, T.-K., and Puchta, H. (2021). Novel CRISPR/Cas applications in plants: from prime editing to chromosome engineering. Transgenic Res. doi: 10.1007/ s11248-021-00238-x [Epub ahead of print].

Huang, S., Weigel, D., Beachy, R. N., and Li, J. (2016). A proposed regulatory framework for genome-edited crops. Nat. Genet. 48, 109-111. doi: 10.1038/ ng.3484

Ito, Y., Nishizawa-Yokoi, A., Endo, M., Mikami, M., and Toki, S. (2015). CRISPR/ Cas9-mediated mutagenesis of the RIN locus that regulates tomato fruit ripening. Biochem. Biophys. Res. Commun. 467, 76-82. doi: 10.1016/j.bbrc. 2015.09.117

Jankele, R., and Svoboda, P. (2014). TAL effectors: tools for DNA targeting. Brief. Funct. Genomics 13, 409-419. doi: 10.1093/bfgp/elu013

Jankowicz-Cieslak, J., Mba, C., and Till, B. J. (2017). "Mutagenesis for crop breeding and functional genomics" in Biotechnologies for Plant Mutation 
Breeding: Protocols. eds. J. Jankowicz-Cieslak, T. H. Tai, J. Kumlehn and B. J. Till (Cham: Springer International Publishing), 3-18.

Jat, S. K., Bhattacharya, J., and Sharma, M. K. (2020). Nanomaterial based gene delivery: a promising method for plant genome engineering. J. Mater. Chem. B 8, 4165-4175. doi: 10.1039/D0TB00217H

Jeong, S. Y., Ahn, H., Ryu, J., Oh, Y., Sivanandhan, G., Won, K.-H., et al. (2019). Generation of early-flowering Chinese cabbage (Brassica rapa spp. pekinensis) through CRISPR/Cas9-mediated genome editing. Plant Biotechnol. Rep. 13, 491-499. doi: 10.1007/s11816-019-00566-9

Jiang, N., Zhang, C., Liu, J. Y., Guo, Z. H., Zhang, Z. Y., Han, C. G., et al. (2019). Development of beet necrotic yellow vein virus-based vectors for multiple-gene expression and guide RNA delivery in plant genome editing. Plant Biotechnol. J. 17, 1302-1315. doi: 10.1111/pbi.13055

Jin, S., Fei, H., Zhu, Z., Luo, Y., Liu, J., Gao, S., et al. (2020). Rationally designed $\mathrm{APOBEC3B}$ cytosine base editors with improved specificity. Mol. Cell 79, 728.e726-740.e726. doi: 10.1016/j.molcel.2020.07.005

Jinek, M., Chylinski, K., Fonfara, I., Hauer, M., Doudna, J. A., and Charpentier, E. (2012). A programmable dual-RNA-guided DNA endonuclease in adaptive bacterial immunity. Science 337, 816-821. doi: 10.1126/science.1225829

Jouanin, A., Boyd, L., Visser, R. G. F., and Smulders, M. J. M. (2018). Development of wheat with hypoimmunogenic gluten obstructed by the gene editing policy in Europe. Front. Plant Sci. 9:1523. doi: 10.3389/fpls.2018.01523

Joung, J. K., and Sander, J. D. (2013). TALENs: a widely applicable technology for targeted genome editing. Nat. Rev. Mol. Cell Biol. 14, 49-55. doi: 10.1038/nrm3486

Jung, Y.-J., Nogoy, F. M., Lee, S.-K., Cho, Y.-G., and Kang, K.-K. (2018). Application of ZFN for site directed mutagenesis of rice SSIVa gene. Biotechnol. Bioprocess Eng. 23, 108-115. doi: 10.1007/s12257-017-0420-9

Kang, B.-C., Yun, J.-Y., Kim, S.-T., Shin, Y., Ryu, J., Choi, M., et al. (2018). Precision genome engineering through adenine base editing in plants. Nat. Plants 4, 427-431. doi: 10.1038/s41477-018-0178-x

Karkute, S. G., Singh, A. K., Gupta, O. P., Singh, P. M., and Singh, B. (2017). CRISPR/Cas9 mediated genome engineering for improvement of horticultural crops. Front. Plant Sci. 8:1635. doi: 10.3389/fpls.2017.01635

Khatodia, S., Bhatotia, K., and Tuteja, N. (2017). Development of CRISPR/ Cas9 mediated virus resistance in agriculturally important crops. Bioengineered 8, 274-279. doi: 10.1080/21655979.2017.1297347

Kim, H., and Kim, J.-S. (2014). A guide to genome engineering with programmable nucleases. Nat. Rev. Genet. 15, 321-334. doi: 10.1038/nrg3686

Kim, H., Kim, S. T., Ryu, J., Choi, M. K., Kweon, J., Kang, B. C., et al. (2016). A simple, flexible and high-throughput cloning system for plant genome editing via CRISPR-Cas system. J. Integr. Plant Biol. 58, 705-712. doi: 10.1111/ jipb.12474

Kim, Y. G., Cha, J., and Chandrasegaran, S. (1996). Hybrid restriction enzymes: zinc finger fusions to Fok I cleavage domain. Proc. Natl. Acad. Sci. U. S. A. 93, 1156-1160. doi: 10.1073/pnas.93.3.1156

Kleinstiver, B. P., Sousa, A. A., Walton, R. T., Tak, Y. E., Hsu, J. Y., Clement, K., et al. (2019). Engineered CRISPR-Cas12a variants with increased activities and improved targeting ranges for gene, epigenetic and base editing. Nat. Biotechnol. 37, 276-282. doi: 10.1038/s41587-018-0011-0

Kleter, G. A., Kuiper, H. A., and Kok, E. J. (2019). Gene-edited crops: towards a harmonized safety assessment. Trends Biotechnol. 37, 443-447. doi: 10.1016/j. tibtech.2018.11.014

Langner, T., Kamoun, S., and Belhaj, K. (2018). CRISPR Crops: plant genome editing toward disease resistance. Annu. Rev. Phytopathol. 56, 479-512. doi: 10.1146/annurev-phyto-080417-050158

Lee, J., Chung, J.-H., Kim, H. M., Kim, D.-W., and Kim, H. (2016). Designed nucleases for targeted genome editing. Plant Biotechnol. J. 14, 448-462. doi: 10.1111/pbi.12465

Lee, M. H., Lee, J., Choi, S. A., Kim, Y.-S., Koo, O., Choi, S. H., et al. (2020). Efficient genome editing using CRISPR-Cas9 RNP delivery into cabbage protoplasts via electro-transfection. Plant Biotechnol. Rep. 14, 695-702. doi: 10.1007/s11816-020-00645-2

Lei, J., Dai, P., Li, J., Yang, M., Li, X., Zhang, W., et al. (2021). Tissue-specific CRISPR/Cas9 system of cotton pollen with GhPLIMP2b and GhMYB24 promoters. J. Plant Bio. 64, 13-21. doi: 10.1007/s12374-020-09272-4

Li, C., Li, W., Zhou, Z., Chen, H., Xie, C., and Lin, Y. (2020a). A new rice breeding method: CRISPR/Cas9 system editing of the Xa13 promoter to cultivate transgene-free bacterial blight-resistant rice. Plant Biotechnol. J. 18, 313-315. doi: $10.1111 /$ pbi.13217
Li, C., Zhang, R., Meng, X., Chen, S., Zong, Y., Lu, C., et al. (2020b). Targeted, random mutagenesis of plant genes with dual cytosine and adenine base editors. Nat. Biotechnol. 38, 875-882. doi: 10.1038/s41587-019-0393-7

Li, C., Zong, Y., Wang, Y., Jin, S., Zhang, D., Song, Q., et al. (2018a). Expanded base editing in rice and wheat using a Cas9-adenosine deaminase fusion. Genome Biol. 19:59. doi: 10.1186/s13059-018-1443-z

Li, J., Manghwar, H., Sun, L., Wang, P., Wang, G., Sheng, H., et al. (2019). Whole genome sequencing reveals rare off-target mutations and considerable inherent genetic or/and somaclonal variations in CRISPR/Cas9-edited cotton plants. Plant Biotechnol. J. 17, 858-868. doi: 10.1111/pbi.13020

Li, J., Meng, X., Zong, Y., Chen, K., Zhang, H., Liu, J., et al. (2016a). Gene replacements and insertions in rice by intron targeting using CRISPR-Cas9. Nat. Plants 2:16139. doi: 10.1038/nplants.2016.139

Li, M., Li, X., Zhou, Z., Wu, P., Fang, M., Pan, X., et al. (2016b). Reassessment of the four yield-related genes Gn1a, DEP1, GS3, and IPA1 in rice using a CRISPR/Cas9 system. Front. Plant Sci. 7:377. doi: 10.3389/fpls.2016. 00377

Li, T., Liu, B., Spalding, M. H., Weeks, D. P., and Yang, B. (2012). Highefficiency TALEN-based gene editing produces disease-resistant rice. Nat. Biotechnol. 30, 390-392. doi: 10.1038/nbt.2199

Li, T., Yang, X., Yu, Y., Si, X., Zhai, X., Zhang, H., et al. (2018b). Domestication of wild tomato is accelerated by genome editing. Nat. Biotechnol. 36, 1160-1163. doi: $10.1038 /$ nbt. 4273

Liang, Z., Chen, K., Li, T., Zhang, Y., Wang, Y., Zhao, Q., et al. (2017). Efficient DNA-free genome editing of bread wheat using CRISPR/Cas9 ribonucleoprotein complexes. Nat. Commun. 8:14261. doi: 10.1038/ncomms14261

Lin, Q., Zong, Y., Xue, C., Wang, S., Jin, S., Zhu, Z., et al. (2020). Prime genome editing in rice and wheat. Nat. Biotechnol. 38, 582-585. doi: 10.1038/ s41587-020-0455-x

Liu, G., Qi, Y., and Zhang, T. (2021a). "Analysis of off-target mutations in CRISPR-Edited rice plants using whole-genome sequencing" in Rice Genome Engineering and Gene Editing: Methods and Protocols. eds. A. Bandyopadhyay and R. Thilmony (New York, NY: Springer US), 145-172.

Liu, J., Fernie, A. R., and Yan, J. (2020). The past, present, and future of maize improvement: domestication, genomics, and functional genomic routes toward crop enhancement. Plant Comm. 1:100010. doi: 10.1016/j.xplc.2019.100010

Liu, J., Nannas, N. J., Fu, F.-F., Shi, J., Aspinwall, B., Parrott, W. A., et al. (2019). Genome-scale sequence disruption following biolistic transformation in rice and maize. Plant Cell 31, 368-383. doi: 10.1105/tpc.18.00613

Liu, M., Zhang, W., Xin, C., Yin, J., Shang, Y., Ai, C., et al. (2021b). Global detection of DNA repair outcomes induced by CRISPR-Cas9. bioRxiv [Preprint]. doi: 10.1101/2021.02.15.431335

Lu, Y., and Zhu, J. K. (2017). Precise editing of a target base in the rice genome using a modified CRISPR/Cas9 system. Mol. Plant 10, 523-525. doi: 10.1016/j.molp.2016.11.013

Ma, C., Liu, M., Li, Q., Si, J., Ren, X., and Song, H. (2019a). Efficient BoPDS gene editing in cabbage by the CRISPR/Cas9 system. Hortic. Plant J. 5, 164-169. doi: 10.1016/j.hpj.2019.04.001

Ma, C., Zhu, C., Zheng, M., Liu, M., Zhang, D., Liu, B., et al. (2019b). CRISPR/ Cas9-mediated multiple gene editing in Brassica oleracea var. capitata using the endogenous tRNA-processing system. Horti. Res. 6:20. doi: 10.1038/ s41438-018-0107-1

Ma, X., Zhang, X., Liu, H., and Li, Z. (2020). Highly efficient DNA-free plant genome editing using virally delivered CRISPR-Cas9. Nat. Plants 6, 773-779. doi: 10.1038/s41477-020-0704-5

Macovei, A., Sevilla, N. R., Cantos, C., Jonson, G. B., Slamet-Loedin, I., Čermák, T., et al. (2018). Novel alleles of rice eIF4G generated by CRISPR/ Cas9-targeted mutagenesis confer resistance to rice tungro spherical virus. Plant Biotechnol. J. 16, 1918-1927. doi: 10.1111/pbi.12927

Mahas, A., Aman, R., and Mahfouz, M. (2019). CRISPR-Cas13d mediates robust RNA virus interference in plants. Genome Biol. 20:263. doi: 10.1186/ s13059-019-1881-2

Maher, M. F., Nasti, R. A., Vollbrecht, M., Starker, C. G., Clark, M. D., and Voytas, D. F. (2020). Plant gene editing through de novo induction of meristems. Nat. Biotechnol. 38, 84-89. doi: 10.1038/s41587-019-0337-2

Maioli, A., Gianoglio, S., Moglia, A., Acquadro, A., Valentino, D., Milani, A. M., et al. (2020). Simultaneous CRISPR/Cas9 editing of three PPO genes reduces fruit flesh browning in Solanum melongena L. Front. Plant Sci. 11:607161. doi: $10.3389 /$ fpls.2020.607161 
Makarova, K. S., Wolf, Y. I., Alkhnbashi, O. S., Costa, F., Shah, S. A., Saunders, S. J., et al. (2015). An updated evolutionary classification of CRISPR-Cas systems. Nat. Rev. Microbiol. 13, 722-736. doi: 10.1038/nrmicro3569

Mali, P., Yang, L., Esvelt, K. M., Aach, J., Guell, M., DiCarlo, J. E., et al. (2013). RNA-guided human genome engineering via Cas9. Science 339, 823-826. doi: 10.1126/science.1232033

Maoto, M. M., Beswa, D., and Jideani, A. I. O. (2019). Watermelon as a potential fruit snack. Int. J. Food Prop. 22, 355-370. doi: 10.1080/ 10942912.2019.1584212

Martin, A., Troadec, C., Boualem, A., Rajab, M., Fernandez, R., Morin, H., et al. (2009). A transposon-induced epigenetic change leads to sex determination in melon. Nature 461, 1135-1138. doi: 10.1038/nature08498

Mei, Y., Beernink, B. M., Ellison, E. E., Konečná, E., Neelakandan, A. K., Voytas, D. F., et al. (2019). Protein expression and gene editing in monocots using foxtail mosaic virus vectors. Plant Direct. 3:e00181. doi: 10.1002/pld3.181

Miller, J., McLachlan, A. D., and Klug, A. (1985). Repetitive zinc-binding domains in the protein transcription factor IIIA from Xenopus oocytes. EMBO J. 4, 1609-1614. doi: 10.1002/j.1460-2075.1985.tb03825.x

Miller, V., Mente, A., Dehghan, M., Rangarajan, S., Zhang, X., Swaminathan, S., et al. (2017). Fruit, vegetable, and legume intake, and cardiovascular disease and deaths in 18 countries (PURE): a prospective cohort study. Lancet 390, 2037-2049. doi: 10.1016/S0140-6736(17)32253-5

Minkenberg, B., Wheatley, M., and Yang, Y. (2017). "Chapter seven - CRISPR/ Cas9-enabled multiplex genome editing and its application" in Progress in Molecular Biology and Translational Science. eds. D. P. Weeks and B. Yang (Massachusetts: Academic Press), 111-132.

Modrzejewski, D., Hartung, F., Lehnert, H., Sprink, T., Kohl, C., Keilwagen, J., et al. (2020). Which factors affect the occurrence of off-target effects caused by the use of CRISPR/Cas: a systematic review in plants. Front. Plant Sci. 11:574959. doi: 10.3389/fpls.2020.574959

Muller, H. J. (1927). Artificial transmutation of the gene. Science 66, 84-87. doi: $10.1126 /$ science.66.1699.84

Mushtaq, M., Bhat, J. A., Mir, Z. A., Sakina, A., Ali, S., Singh, A. K., et al. (2018). CRISPR/Cas approach: a new way of looking at plant-abiotic interactions. J. Plant Physiol. 224-225, 156-162. doi: 10.1016/j.jplph.2018.04.001

Nakayasu, M., Akiyama, R., Lee, H. J., Osakabe, K., Osakabe, Y., Watanabe, B., et al. (2018). Generation of $\alpha$-solanine-free hairy roots of potato by CRISPR/ Cas9 mediated genome editing of the St16DOX gene. Plant Physiol. Biochem. 131, 70-77. doi: 10.1016/j.plaphy.2018.04.026

Ninfali, P., Mea, G., Giorgini, S., Rocchi, M., and Bacchiocca, M. (2005). Antioxidant capacity of vegetables, spices and dressings relevant to nutrition. Br. J. Nutr. 93, 257-266. doi: 10.1079/BJN20041327

Nunez de Caceres Gonzalez, F. F., and De la Mora Franco, D. (2020). "Vegetable crop improvement using CRISPR/Cas9" in CRISPR/Cas Genome Editing: Strategies And Potential For Crop Improvement. eds. A. Bhattacharya, V. Parkhi and B. Char (Cham: Springer International Publishing), 119-129.

Oh, Y., Lee, B., Kim, H., and Kim, S.-G. (2020). A multiplex guide RNA expression system and its efficacy for plant genome engineering. Plant Methods 16:37. doi: 10.1186/s13007-020-00580-x

Peer, R., Rivlin, G., Golobovitch, S., Lapidot, M., Gal-On, A., Vainstein, A., et al. (2015). Targeted mutagenesis using zinc-finger nucleases in perennial fruit trees. Planta 241, 941-951. doi: 10.1007/s00425-014-2224-x

Pennisi, E. (2013). The CRISPR craze. Science 341, 833-836. doi: 10.1126/ science.341.6148.833

Petolino, J. F. (2015). Genome editing in plants via designed zinc finger nucleases. In Vitro Cell Dev. Biol. Plant 51, 1-8. doi: 10.1007/s11627-015-9663-3

Radhamony, R. N., Mohan Prasad, A., and Srinivasan, R. (2005). T-DNA insertional mutagenesis in Arabidopsis: a tool for functional genomics. Electron. J. Biotechnol. 8, 82-106. doi: 10.2225/vol8-issue1-fulltext-4

Ran, F. A., Cong, L., Yan, W. X., Scott, D. A., Gootenberg, J. S., Kriz, A. J., et al. (2015). In vivo genome editing using Staphylococcus aureus Cas9. Nature 520, 186-191. doi: 10.1038/nature14299

Ren, B., Yan, F., Kuang, Y., Li, N., Zhang, D., Zhou, X., et al. (2018). Improved base editor for efficiently inducing genetic variations in rice with CRISPR/ Cas9-guided hyperactive hAID mutant. Mol. Plant 11, 623-626. doi: 10.1016/j. molp.2018.01.005

Ren, Q., Sretenovic, S., Liu, S., Tang, X., Huang, L., He, Y., et al. (2021). PAM-less plant genome editing using a CRISPR-SpRY toolbox. Nat. Plants 7, 25-33. doi: 10.1038/s41477-020-00827-4
Richter, M. F., Zhao, K. T., Eton, E., Lapinaite, A., Newby, G. A., Thuronyi, B. W., et al. (2020). Phage-assisted evolution of an adenine base editor with improved cas domain compatibility and activity. Nat. Biotechnol. 38, 883-891. doi: $10.1038 / \mathrm{s} 41587-020-0453-\mathrm{z}$

Robinson, R. W. (2000). Rationale and methods for producing hybrid cucurbit seed. J. New Seeds 1, 1-47. doi: 10.1300/J153v01n03_01

Rodríguez-Leal, D., Lemmon, Z. H., Man, J., Bartlett, M. E., and Lippman, Z. B. (2017). Engineering quantitative trait variation for crop improvement by genome editing. Cell 171, 470.e478-480.e478. doi: 10.1016/j.cell.2017. 08.030

Sakuma, T., and Yamamoto, T. (2017). Current overview of TALEN construction systems. Methods Mol. Biol. 1630, 25-36. doi: 10.1007/978-1-4939-7128-2_2

Santillán Martínez, M. I., Bracuto, V., Koseoglou, E., Appiano, M., Jacobsen, E., Visser, R. G. F., et al. (2020). CRISPR/Cas 9 -targeted mutagenesis of the tomato susceptibility gene PMR4 for resistance against powdery mildew. BMC Plant Biol. 20:284. doi: 10.1186/s12870-020-02497-y

Sawai, S., Ohyama, K., Yasumoto, S., Seki, H., Sakuma, T., Yamamoto, T., et al. (2014). Sterol side chain reductase 2 is a key enzyme in the biosynthesis of cholesterol, the common precursor of toxic steroidal glycoalkaloids in potato. Plant Cell 26, 3763-3774. doi: 10.1105/tpc.114.130096

Schmidt, C., Fransz, P., Rönspies, M., Dreissig, S., Fuchs, J., Heckmann, S., et al. (2020). Changing local recombination patterns in Arabidopsis by CRISPR/Cas mediated chromosome engineering. Nat. Commun. 11:4418. doi: $10.1038 / \mathrm{s} 41467-020-18277-\mathrm{z}$

Schmidt, C., Pacher, M., and Puchta, H. (2019). Efficient induction of heritable inversions in plant genomes using the CRISPR/Cas system. Plant J. 98, 577-589. doi: $10.1111 /$ tpj.14322

Septembre-Malaterre, A., Remize, F., and Poucheret, P. (2018). Fruits and vegetables, as a source of nutritional compounds and phytochemicals: changes in bioactive compounds during lactic fermentation. Food Res. Int. 104, 86-99. doi: 10.1016/j.foodres.2017.09.031

Serafini, M., Bellocco, R., Wolk, A., and Ekström, A. M. (2002). Total antioxidant potential of fruit and vegetables and risk of gastric cancer. Gastroenterology 123, 985-991. doi: 10.1053/gast.2002.35957

Shan, Q., Wang, Y., Li, J., Zhang, Y., Chen, K., Liang, Z., et al. (2013). Targeted genome modification of crop plants using a CRISPR-Cas system. Nat. Biotechnol. 31, 686-688. doi: 10.1038/nbt.2650

Shelake, R. M., Pramanik, D., and Kim, J. Y. (2019). Exploration of plantmicrobe interactions for sustainable agriculture in CRISPR era. Microorganisms 7:269. doi: 10.3390/microorganisms7080269

Shew, A. M., Nalley, L. L., Snell, H. A., Nayga, R. M., and Dixon, B. L. (2018). CRISPR versus GMOs: public acceptance and valuation. Glob. Food Sec. 19, 71-80. doi: 10.1016/j.gfs.2018.10.005

Shimatani, Z., Kashojiya, S., Takayama, M., Terada, R., Arazoe, T., Ishii, H., et al. (2017). Targeted base editing in rice and tomato using a CRISPR-Cas 9 cytidine deaminase fusion. Nat. Biotechnol. 35, 441-443. doi: 10.1038/nbt.3833

Shu, Q. Y., Forster, B. P., and Nakagawa, H. (2012). Plant Mutation Breeding and Biotechnology (Wallingford: CABI).

Shukla, V. K., Doyon, Y., Miller, J. C., DeKelver, R. C., Moehle, E. A., and Worden, S. E., et al. (2009). Precise genome modification in the crop species Zea mays using zinc-finger nucleases. Nature 459, 437-441. doi:10.1038/ nature07992

Stadler, L. J. (1928a). Genetic effects of X-rays in maize. Proc. Natl. Acad. Sci. U. S. A. $14,69-75$. doi: $10.1073 /$ pnas.14.1.69

Stadler, L. J. (1928b). Mutations in barley induced by X-rays and radium. Science 68, 186-187. doi: 10.1126/science.68.1756.186

Sun, B., Jiang, M., Zheng, H., Jian, Y., Huang, W.-L., Yuan, Q., et al. (2020). Color-related chlorophyll and carotenoid concentrations of Chinese kale can be altered through CRISPR/Cas9 targeted editing of the carotenoid isomerase gene BoaCRTISO. Hortic. Res. 7:161. doi: 10.1038/s41438-020-00379-w

Sun, B., Zheng, A., Jiang, M., Xue, S., Yuan, Q., Jiang, L., et al. (2018). CRISPR/ Cas9-mediated mutagenesis of homologous genes in Chinese kale. Sci. Rep. 8:16786. doi: $10.1038 / \mathrm{s} 41598-018-34884-9$

Svitashev, S., Schwartz, C., Lenderts, B., Young, J. K., and Mark Cigan, A. (2016). Genome editing in maize directed by CRISPR-Cas9 ribonucleoprotein complexes. Nat. Commun. 7:13274. doi: 10.1038/ncomms13274

Swinnen, G., Goossens, A., and Pauwels, L. (2016). Lessons from domestication: targeting Cis-regulatory elements for crop improvement. Trends Plant Sci. 21, 506-515. doi: 10.1016/j.tplants.2016.01.014 
Symington, L. S., and Gautier, J. (2011). Double-strand break end resection and repair pathway choice. Annu. Rev. Genet. 45, 247-271. doi: 10.1146/ annurev-genet-110410-132435

Tang, L., Mao, B., Li, Y., Lv, Q., Zhang, L., Chen, C., et al. (2017). Knockout of OsNramp5 using the CRISPR/Cas9 system produces low Cd-accumulating indica rice without compromising yield. Sci. Rep. 7:14438. doi: 10.1038/ s41598-017-14832-9

Tatlioglu, T. (1993). “13 - Cucumber: Cucumis sativus L,. in Genetic Improvement of Vegetable Crops. eds. G. Kallo and B. O. Bergh (Amsterdam: Pergamon), 197-234.

Tian, S., Jiang, L., Cui, X., Zhang, J., Guo, S., Li, M., et al. (2018). Engineering herbicide-resistant watermelon variety through CRISPR/Cas9-mediated baseediting. Plant Cell Rep. 37, 1353-1356. doi: 10.1007/s00299-018-2299-0

Tian, S., Jiang, L., Gao, Q., Zhang, J., Zong, M., Zhang, H., et al. (2017). Efficient CRISPR/Cas9-based gene knockout in watermelon. Plant Cell Rep. 36, 1-8. doi: 10.1007/s00299-016-2089-5

Till, B. J., Cooper, J., Tai, T. H., Colowit, P., Greene, E. A., Henikoff, S., et al. (2007). Discovery of chemically induced mutations in rice by TILLING. BMC Plant Biol. 7:19. doi: 10.1186/1471-2229-7-19

Till, B. J., Reynolds, S. H., Weil, C., Springer, N., Burtner, C., Young, K., et al. (2004). Discovery of induced point mutations in maize genes by TILLING. BMC Plant Biol. 4:12. doi: 10.1186/1471-2229-4-12

Tomlinson, L., Yang, Y., Emenecker, R., Smoker, M., Taylor, J., Perkins, S., et al. (2019). Using CRISPR/Cas9 genome editing in tomato to create a gibberellin-responsive dominant dwarf DELLA allele. Plant Biotechnol. J. 17, 132-140. doi: 10.1111/pbi.12952

Urnov, F. D., Rebar, E. J., Holmes, M. C., Zhang, H. S., and Gregory, P. D. (2010). Genome editing with engineered zinc finger nucleases. Nat. Rev. Genet. 11, 636-646. doi: 10.1038/nrg2842

Valton, J., Dupuy, A., Daboussi, F., Thomas, S., Maréchal, A., Macmaster, R., et al. (2012). Overcoming transcription activator-like effector (TALE) DNA binding domain sensitivity to cytosine methylation. J. Biol. Chem. 287, 38427-38432. doi: 10.1074/jbc.C112.408864

Vats, S., Kumawat, S., Kumar, V., Patil, G. B., Joshi, T., Sonah, H., et al. (2019). Genome editing in plants: exploration of technological advancements and challenges. Cell 8:1386. doi: 10.3390/cells8111386

Vejlupkova, Z., Warman, C., Sharma, R., Scheller, H. V., Mortimer, J. C., and Fowler, J. E. (2020). No evidence for transient transformation via pollen magnetofection in several monocot species. bioRxiv 6, 1323-1324. doi: 10.1101/2020.05.01.071266

Wang, L., Chen, L., Li, R., Zhao, R., Yang, M., Sheng, J., et al. (2017). Reduced drought tolerance by CRISPR/Cas9-mediated SIMAPK3 mutagenesis in tomato plants. J. Agric. Food Chem. 65, 8674-8682. doi: 10.1021/acs.jafc.7b02745

Wang, Y., Cheng, X., Shan, Q., Zhang, Y., Liu, J., Gao, C., et al. (2014). Simultaneous editing of three homoeoalleles in hexaploid bread wheat confers heritable resistance to powdery mildew. Nat. Biotechnol. 32, 947-951. doi: 10.1038/nbt.2969

Weinthal, D., Tovkach, A., Zeevi, V., and Tzfira, T. (2010). Genome editing in plant cells by zinc finger nucleases. Trends Plant Sci. 15, 308-321. doi: 10.1016/j.tplants.2010.03.001

WHO (2003). "Diet, nutrition and the prevention of chronic diseases," in World Health Organization Technical Report Series; May 29, 2003 (Geneva: World Health Organization).

Wolter, F., and Puchta, H. (2018). Application of CRISPR/Cas to understand Cis- and trans-regulatory elements in plants. Methods Mol. Biol. 1830, 23-40. doi: 10.1007/978-1-4939-8657-6_2

Woo, J. W., Kim, J., Kwon, S. I., Corvalán, C., Cho, S. W., Kim, H., et al. (2015). DNA-free genome editing in plants with preassembled CRISPR-Cas9 ribonucleoproteins. Nat. Biotechnol. 33, 1162-1164. doi: 10.1038/nbt.3389

Wunderlich, S., and Gatto, K. A. (2015). Consumer perception of genetically modified organisms and sources of information. Adv. Nutr. 6, 842-851. doi: 10.3945/an.115.008870

Xu, R., Yang, Y., Qin, R., Li, H., Qiu, C., Li, L., et al. (2016). Rapid improvement of grain weight via highly efficient CRISPR/Cas9-mediated multiplex genome editing in rice. J. Genet. Genomics 43, 529-532. doi: 10.1016/j.jgg.2016.07.003

Yamada, M., Watanabe, Y., Gootenberg, J. S., Hirano, H., Ran, F. A., Nakane, T., et al. (2017). Crystal structure of the minimal Cas9 from campylobacter jejuni reveals the molecular diversity in the CRISPR-Cas9 systems. Mol. Cell 65, 1109.e1103-1121.e1103. doi: 10.1016/j.molcel.2017.02.007

Yin, K., Han, T., Liu, G., Chen, T., Wang, Y., Yu, A. Y. L., et al. (2015). A geminivirus-based guide RNA delivery system for CRISPR/Cas9 mediated plant genome editing. Sci. Rep. 5:14926. doi: 10.1038/srep14926

Yoshida, H., Hirano, K., Sato, T., Mitsuda, N., Nomoto, M., Maeo, K., et al. (2014). DELLA protein functions as a transcriptional activator through the DNA binding of the indeterminate domain family proteins. Proc. Natl. Acad. Sci. 111, 7861-7866. doi: 10.1073/pnas.1321669111

Yu, Q., and Powles, S. B. (2014). Resistance to AHAS inhibitor herbicides: current understanding. Pest Manag. Sci. 70, 1340-1350. doi: 10.1002/ ps.3710

Yu, W., Wang, L., Zhao, R., Sheng, J., Zhang, S., Li, R., et al. (2019). Knockout of SIMAPK3 enhances tolerance to heat stress involving ROS homeostasis in tomato plants. BMC Plant Biol. 19:354. doi: 10.1186/ s12870-019-1939-z

Zetsche, B., Gootenberg, J. S., Abudayyeh, O. O., Slaymaker, I. M., Makarova, K. S., Essletzbichler, P., et al. (2015). Cpf1 is a single RNA-guided endonuclease of a class 2 CRISPR-Cas system. Cell 163, 759-771. doi: 10.1016/j. cell.2015.09.038

Zhang, C., Liu, C., Weng, J., Cheng, B., Liu, F., Li, X., et al. (2017). Creation of targeted inversion mutations in plants using an RNA-guided endonuclease. Crop J. 5, 83-88. doi: 10.1016/j.cj.2016.08.001

Zhang, H., Si, X., Ji, X., Fan, R., Liu, J., Chen, K., et al. (2018a). Genome editing of upstream open reading frames enables translational control in plants. Nat. Biotechnol. 36, 894-898. doi: 10.1038/nbt.4202

Zhang, J., Guo, S., Ji, G., Zhao, H., Sun, H., Ren, Y., et al. (2020a). A unique chromosome translocation disrupting ClWIP1 leads to gynoecy in watermelon. Plant J. 101, 265-277. doi: 10.1111/tpj.14537

Zhang, J., Zhang, H., Botella, J. R., and Zhu, J.-K. (2018b). Generation of new glutinous rice by CRISPR/Cas9-targeted mutagenesis of the Waxy gene in elite rice varieties. J. Integr. Plant Biol. 60, 369-375. doi: 10.1111/jipb. 12620

Zhang, M., Liu, Q., Yang, X., Xu, J., Liu, G., Yao, X., et al. (2020b). CRISPR/ Cas9-mediated mutagenesis of Clpsk1 in watermelon to confer resistance to Fusarium oxysporum f.sp. niveum. Plant Cell Rep. 39, 589-595. doi: 10.1007/s00299-020-02516-0

Zhang, Y., Pribil, M., Palmgren, M., and Gao, C. (2020c). A CRISPR way for accelerating improvement of food crops. Nat. Food 1, 200-205. doi: 10.1038/ s43016-020-0051-8

Zhao, H., and Wolt, J. D. (2017). Risk associated with off-target plant genome editing and methods for its limitation. Emerg. Top Life Sci. 1, 231-240. doi: 10.1042/ETLS20170037

Zhao, X., Meng, Z., Wang, Y., Chen, W., Sun, C., Cui, B., et al. (2017). Pollen magnetofection for genetic modification with magnetic nanoparticles as gene carriers. Nat. Plants 3, 956-964. doi: 10.1038/s41477-017-0063-Z

Zong, Y., Song, Q., Li, C., Jin, S., Zhang, D., Wang, Y., et al. (2018). Efficient C-to-T base editing in plants using a fusion of nCas9 and human APOBEC3A. Nat. Biotechnol. 36, 950-953. doi: 10.1038/nbt.4261

Zong, Y., Wang, Y., Li, C., Zhang, R., Chen, K., Ran, Y., et al. (2017). Precise base editing in rice, wheat and maize with a Cas9-cytidine deaminase fusion. Nat. Biotechnol. 35, 438-440. doi: 10.1038/nbt.3811

Zsögön, A., Čermák, T., Naves, E. R., Notini, M. M., Edel, K. H., Weinl, S., et al. (2018). De novo domestication of wild tomato using genome editing. Nat. Biotechnol. 36, 1211-1216. doi: 10.1038/nbt.4272

Conflict of Interest: The authors declare that the research was conducted in the absence of any commercial or financial relationships that could be construed as a potential conflict of interest.

Copyright (C) 2021 Kim, Kang, Yang, Cho, Schafleitner, Lee and Jang. This is an open-access article distributed under the terms of the Creative Commons Attribution License (CC BY). The use, distribution or reproduction in other forums is permitted, provided the original author(s) and the copyright owner(s) are credited and that the original publication in this journal is cited, in accordance with accepted academic practice. No use, distribution or reproduction is permitted which does not comply with these terms. 